\title{
Control of a 2-DOF manipulator with a flexible forearm
}

\author{
KOH TUCK LYE, H KRISHNAN* and C L TEO \\ Department of Mechanical and Production Engineering, National University \\ of Singapore, 10 Kent Ridge Crescent, Singapore 119260 \\ e-mail: mpehk@nus.sg (H Krishnan)
}

\begin{abstract}
In this paper we present experimental results on the position and vibration control of the end-effector of a 2-DOF parallelogram manipulator with a flexible forearm. A dynamic model of the manipulator is first obtained. Control strategies are implemented to control the manipulator. The first control strategy uses the computed torque method based on a reduced-order dynamic model of the manipulator which is obtained by assuming that all the links are rigid. This method is referred to as the reduced-order computed torque (ROCT) method. Experimental results demonstrate that such a strategy is not good for vibration control of the end-effector of the manipulator. The second control strategy is a state feedback control law designed based on a local linearization of the nonlinear dynamic model. Experimental results show that this control strategy achieves good vibration control of the end-effector of the manipulator. However, since the strategy is based on local linearization, it is valid only in a neighbourhood of the operating point. A hybrid controller that uses the ROCT method for the initial large movement of the manipulator is then implemented. Based on a switching rule, the controller is switched to the state feedback controller based on the linearized model when the manipulator is sufficiently close to the equilibrium state. Experimental results are reported and the successful performance of the controller in dampening out end-point vibrations is demonstrated.
\end{abstract}

Keywords. Two-DOF flexible manipulator; reduced-order computed torque control; vibration control; hybrid controller.

\section{Introduction}

The increasing industrial need for higher productivity requires robots that move accurately and rapidly. In order to achieve this, it is desirable to have robots with light structures that offer high-speed performance and low energy consumption. One way of designing a robot with a light structure is to use slender links. Modelling and control of flexible-link

*Author for correspondence 
manipulators has been a subject of active research in recent years. Flexible-link manipulators have the advantages of being lightweight and have a larger pay-load to weight ratio. They are also easy to fabricate and their design is simple. However the fact that the links are flexible introduces vibrations as the robot arm is in motion. Vibration suppression and position control in flexible-link robot arms is therefore very important. A number of researchers have studied the control design for vibration suppression and position control of a flexible robot arm (see Craig 1981, Book 1984, Cannon \& Schmitz 1984, Hastings \& Book 1986, Sakawa \& Matsuno 1986, Matsuno \& Fukushima 1987, Siciliano \& Book 1988, Wang \& Vidyasagar 1991, Siciliano et al 1992, De Luca \& Siciliano 1993, Aoustin et al 1994, Gross \& Tomizuka 1994, Khorrami et al 1994, Moudgal et al 1994, Vandergrift et al 1994, Zhu et al 1994, Banavar \& Dominic 1995, Lin \& Yih 1996, and references therein). Successful experimental results have also been reported for single-link and two-link flexible robot arms (Cannon \& Schmitz 1984; Hastings \& Book 1986; Sakawa \& Matsuno 1986; Matsuno \& Fukushima 1987; Aoustin et al 1994; Gross \& Tomizuka 1994; Khorrami et al 1994; Moudgal et al 1994; Banavar \& Dominic 1994). Modelling of flexible robot arms has been carried out in many papers (see Book 1984, for e.g.). It has been pointed out (Siciliano \& Book 1988) that, if the links are stiff, one can use singular perturbation methods for the control design for vibration suppression and position control in n-link flexible manipulators. However the control law given by Siciliano \& Book (1988) requires measurements of the link deflection rates which is not easily measurable. The control law is modified as an output feedback strategy by Siciliano et al (1992) and only requires the joint position measurements, joint velocities, and the link deflection, which are obtained easily, say, from strain gauge measurements. An alternative but similar control design method is also proposed by Vandergrift et al (1994) for vibration suppression and position control of n-link flexible manipulators. A method of control design based on a dynamic output feedback compensator is proposed by Craig (1981), which avoids the need for the velocity of the deflection. Sakawa \& Matsuno (1986) derived a dynamic model for a 2DOF parallelogram manipulator and designed a feedback control system for it. This was followed up by Matsuno \& Fukushima (1987) who obtained several satisfactory results for point-to-point position control.

The objective of this paper is to report experimental results on the position and vibration control of the end-effector of a 2-DOF parallelogram manipulator with a flexible forearm. A dynamic model is first derived. Control strategies are implemented on the experimental facility. The first control strategy uses the computed torque method based on a reduced-order dynamic model of the manipulator which is obtained by assuming that all the manipulator links are rigid. This method is referred to as the reducedorder computed torque (ROCT) method. The second control strategy is a state feedback control law designed based on a local linearization of the nonlinear dynamic model of the system. Finally, the third control strategy uses the ROCT control for the initial large movement of the manipulator. The control law is then switched to the state feedback control based on a switching rule. This method is referred to as the hybrid control method.

The paper is organised as follows. Section 2 describes the experimental setup. This is followed by the derivation of the dynamic model of the system in $\S 3$. Using this dynamic model, three different control strategies are developed. Section 4 presents the experimental 


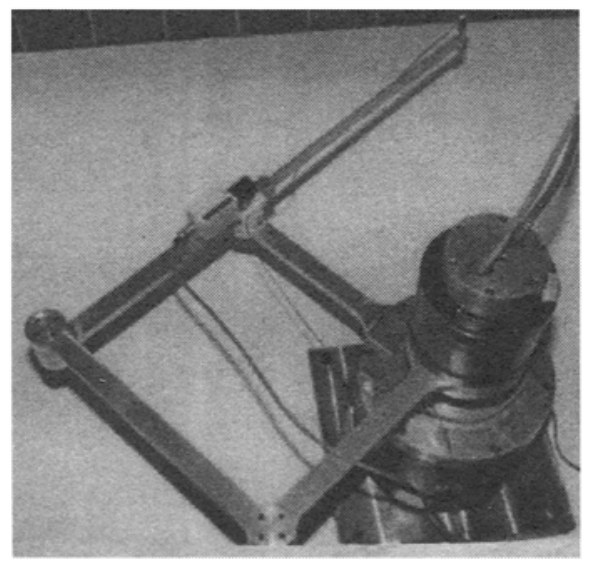

Figure 1. The manipulator with a flexible forearm.

results of the ROCT method. In $\S 5$, the state feedback control law and hybrid control law are discussed and the experimental results are given. The conclusions are presented in $\S 6$.

\section{Experimental setup}

This section provides a brief description of the experimental setup. The objective of this paper is to present experimental results on the position and vibration control of the endeffector of a 2-DOF manipulator with a flexible forearm. Figure 1 shows a picture of the manipulator in the experimental facility.

The manipulator is a 2-DOF parallelogram design similar to the one developed by Mills \& Lokhorst (1993). The last link (forearm) of the robot is flexible. It operates in the horizontal plane and is driven by 2 Dynaserv direct-drive brushless motors so that the problem of friction and backlash are minimized. The characteristics of the motors used in the experimental robot are shown in table 1. Note that the motors have high performance, high torque output which prevents saturation, and high precision encoder resolution. The servo drivers for the motors can be preset to operate in 3 modes of control, namely, position control mode, speed control mode and torque/current control mode. In this experiment we preset the operation of the servo drivers to the torque/current control mode. Thus a voltage between $-8 \mathrm{~V}$ to $8 \mathrm{~V}$ applied to the servo drivers allows the motor to produce a torque between negative maximum torque and positive maximum

Table 1. Motor characteristics.

\begin{tabular}{llll}
\hline & & \multicolumn{2}{c}{ Motor model } \\
\cline { 3 - 4 } Feature & Unit & DM1200A & DM1045B \\
\hline Maximum output torque & $\mathrm{Nm}$ & 200 & 45 \\
Rated speed & $\mathrm{rev} / \mathrm{s}$ & 1.0 & 2.0 \\
Encoder resolution & $\mathrm{p} / \mathrm{rev}$ & 1024000 & 655360 \\
Weight & $\mathrm{kg}$ & 29 & 9.5 \\
Rotor inertia & $\mathrm{kgm}^{2}$ & 0.167 & 0.019 \\
\hline
\end{tabular}




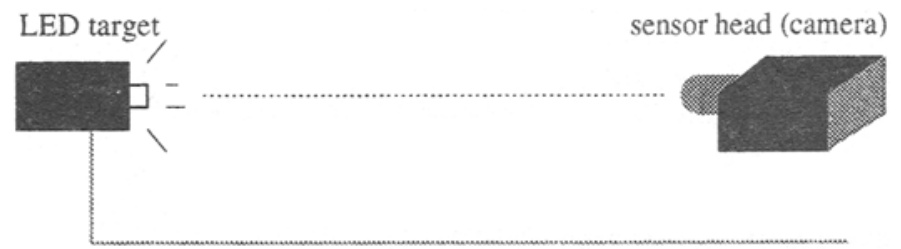

Figure 2. Schematic diagram of an active type PSD.

torque, and the voltage-to-torque relationship is linear. Thus direct torque control is achieved.

In this experiment, measurement of the vibration at the end-effector of the robot is essential. The Hamamatsu C2399-00 position-sensitive detector (PSD) is used for this purpose. This is an active type, opto-electric position sensing unit which measures the position of a single-point of light spot focused on the sensor head. It uses an infrared LED mounted as a target at the tip of the flexible link and the movement of the LED is measured by a sensor camera which is mounted as shown in figure 1. Figure 2 shows the schematic diagram of the active type PSD used in the experiment. The advantage of the C2399-00 PSD is that it uses a non-discrete, silicon PSD for two-dimensional position sensing. The non-discrete nature of the PSD allows for high accuracy measurement of position. The LED is pulse-modulated and optically filtered in the sensor head with a builtin background-light cancellation circuit, making accurate measurements possible even in bright locations. Since it does not require scanning, it provides good speed of response. The advantages of high accuracy measurement and good speed of response make it very suitable for vibration measurement. The rated position resolution of the C2399-00 PSD is $1 / 5000$ and its sampling rate is $312.5 \mathrm{~Hz}$. The sensor head of the PSD is a camera that uses a $\mathrm{C}$-mount lens. The choice of a suitable lens is based on the following 2 factors: (1) the required angle of view which is determined by the dimensions of the scene, and (2) the minimum illumination level of the area to be monitored.

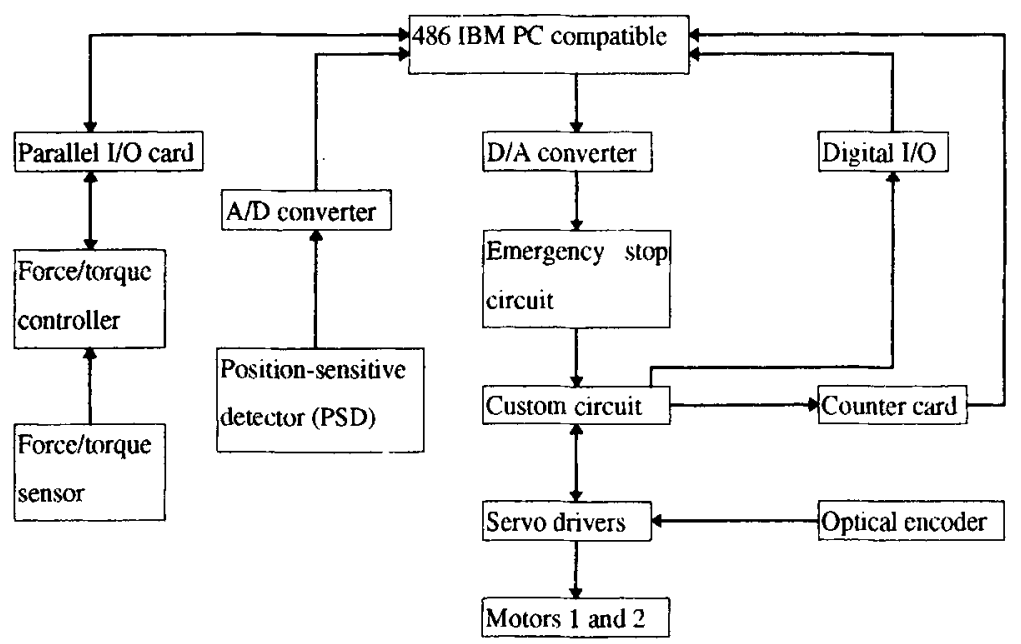

Figure 3. Controller for 2-DOF manipulator with a flexible forearm. 
Figure 3 shows the controller architecture. A quadrature encoder input card along with the motor encoders are used for measurement of link angular displacements. The link angular velocities are then obtained by numerical differentiation. Inputs to this card are conditioned by a 4-stage digital filter. For the experiment, the encoder signals from the 2 motors are input to the card, which provides the digital counts to the computer. The host computer is an IBM PC-compatible machine with a 486DX33 microprocessor with $8 \mathrm{MB}$ RAM. A program written in C-language is used to control the manipulator. A menudriven control panel allows the user to input control parameters such as desired end position of manipulator, sampling rate, and gain constants. At each sampling time, the computer reads the digital input data, performs servo calculations and writes digital output data.

The $\mathrm{D} / \mathrm{A}$ converter for the digital computer operates in bipolar mode and has a 14bit resolution and conversion time less than $42 \mu \mathrm{s}$. The voltage output range of the D/A converter is $-8 \mathrm{~V}+8 \mathrm{~V}$ and its outputs are connected to the servo drivers via an emergency stop circuit and a custom circuit. These are used for controlling the voltages to the two motors. The A/D converters provide analog-to-digital conversion for the analog signal from the PSD. The A/D converter has a 12-bit resolution and best conversion time of $10 \mu \mathrm{s}$. The accuracy and linearity of the converter are both $+/-1$ bit. Besides the $\mathrm{D} / \mathrm{A}$ and A/D converters, the digital $\mathrm{I} / \mathrm{O}$ card is also used as an interface to the computer. They are used to read the status of motors 1 and 2 respectively. An interface to the force/torque sensor is also available as shown in figure 3 . This is used for conducting contact force control experiments using the manipulator.

\section{Dynamic model of the manipulator}

\subsection{Manipulator characteristics and parameters}

Figure 4 shows a schematic diagram of the manipulator with a flexible forearm. It comprises 4 rigid links labelled from links 1 to 4 . Link 4 has an extension $l_{s}$ that is used to clamp flexible link 5. All the links are made of aluminum alloy 5083. A position-sensitive detector

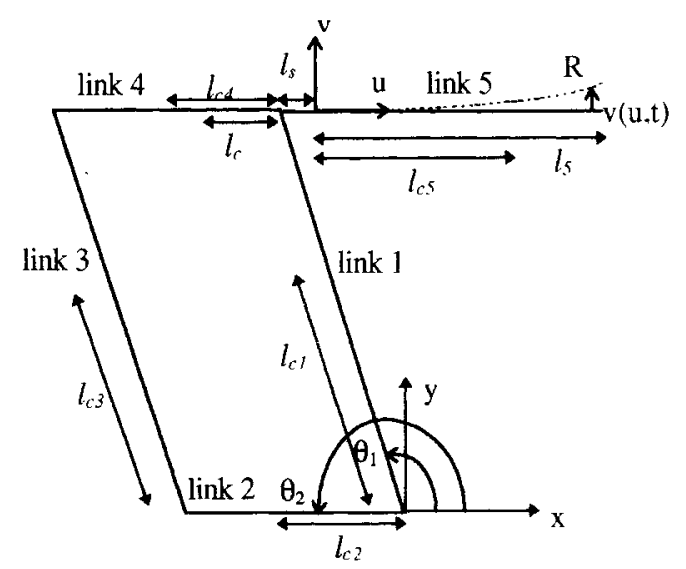

Figure 4. Schematic diagram of the manipulator. 
Table 2. Parameters for camera and LED assemblies.

\begin{tabular}{lcc}
\hline & Camera assembly & LED assembly \\
\hline Length $(\mathrm{m})$ & $l_{c}=0.0645$ & $l_{s}=0.0525, l_{5}=0.4800$ \\
Mass $(\mathrm{kg})$ & $m_{c}=0.209$ & $m_{L}=0.026$ \\
\hline
\end{tabular}

(PSD) is mounted on the manipulator. The PSD consists of a LED assembly which is mounted at the tip of link 5 and a camera assembly mounted at a distance $l_{c}$ from the joint of links 4 and 1 .

Table 2 shows the parameters of the camera assembly and the LED assembly. Table 3 shows the parameters for each link of the parallelogram manipulator.

\subsection{Free transverse vibration of Bernoulli-Euler beam}

In order to derive the dynamic equation of the manipulator, which comprises a flexible forearm labelled as link 5 in figure 4, it is essential to know its vibrational characteristics. Assuming link 5 is a Bernoulli-Euler beam, its vibration is governed by the BernoulliEuler beam equation given by

$$
\frac{\partial^{2}}{\partial u^{2}}\left(E I \frac{\partial^{2} v}{\partial u^{2}}\right)+\rho \frac{\partial^{2} v}{\partial t^{2}}=0
$$

where $0 \leq u \leq l_{5}, \rho$ is the linear density of the beam, $E$ is Young's modulus and $l$ is the cross-sectional area moment of inertia of link 5. The values of $\rho$ and $I$ for link 5 are $0.244 \mathrm{~kg} / \mathrm{m}$ and $6.75 \times 10^{-11} \mathrm{~m}^{4}$ respectively. The assumed-modes method is used in our analysis. The deflection of link 5 is given in separable form as

$$
v(u, t)=\sum_{i=1}^{\infty} \phi_{i}(u) q_{i}(t)
$$

where $\phi_{i}(u)$ is the $i$ th natural mode eigenfunctions, and $q_{i}(t)$ is the time-dependent generalized coordinate. The above equations are solved by approximating the natural modes of flexible link 5 by the natural modes of a uniform clamped-free beam with boundary conditions given by (Craig 1981)

Table 3. Parameters of each individual link.

\begin{tabular}{llllll}
\hline & \multicolumn{1}{c}{ Link 1 } & \multicolumn{1}{c}{ Link 2 } & \multicolumn{1}{c}{ Link 3 } & \multicolumn{1}{c}{ Link 4 } & \multicolumn{1}{c}{ Link 5 } \\
\hline Width $(\mathrm{mm})$ & 30 & 30 & 30 & 30 & 3 \\
Height $(\mathrm{mm})$ & 15 & 15 & 15 & 15 & 30 \\
Length $(\mathrm{m})$ & $l_{1}=0.40$ & $l_{2}=0.35$ & $l_{3}=0.40$ & $l_{4}=0.35$ & $l_{5}=0.48$ \\
Centre of mass $(\mathrm{m})$ & $l_{c 1}=0.058$ & $l_{c 2}=0.090$ & $l_{c 3}=0.195$ & $l_{c 4}=0.173$ & $l_{c 5}=0.293$ \\
Mass $(\mathrm{kg})$ & $m_{1}=2.905$ & $m_{2}=1.505$ & $m_{3}=0.877$ & $m_{4}=0.858$ & $m_{5}=0.117$ \\
Moment of inertia & $I_{1}=0.079$ & $l_{2}=0.031$ & $I_{3}=0.023$ & $l_{4}=0.019$ & $l_{5}=2.24 \times 10^{-3}$ \\
$\quad\left(\mathrm{~kg} / \mathrm{m}^{2}\right)$ & & & & & \\
\hline
\end{tabular}


Table 4. Natural frequencies and related constants of link 5.

\begin{tabular}{llccc}
\hline Mode i & Mode 1 & Mode 2 & Mode 3 & Mode 4 \\
\hline$\beta_{i} l_{5}$ & 1.87510 & 4.69409 & 7.85476 & 10.99554 \\
$\sigma_{i}$ & 0.7341 & 1.0185 & 0.9992 & 1.0000 \\
$\phi_{i}\left(l_{5}\right)$ & 2.00 & -2.00 & 2.00 & -2.00 \\
$\omega_{i}(\mathrm{rad} / \mathrm{s})$ & 69.64 & 436.49 & 1540.89 & 3019.55 \\
$f_{i}(\mathrm{~Hz})$ & 11.08 & 69.47 & 245.24 & 480.58 \\
(for $\left.m_{L}=0\right)$ & & & & \\
\hline
\end{tabular}

$$
\left.v\right|_{u=0}=0,\left.\quad \frac{\partial v}{\partial u}\right|_{u=0}=0,\left.\quad \frac{\partial^{2} v}{\partial u^{2}}\right|_{u=l_{5}}=0,\left.\quad \frac{\partial^{3} v}{\partial u^{3}}\right|_{u=l_{5}}=0 .
$$

The natural mode shape eigenfunctions and the natural frequencies are given by (Craig 1981)

$$
\begin{aligned}
\phi_{i}(u) & =\left[\cosh \beta_{i} u-\cos \beta_{i} u-\sigma_{i}\left(\sinh \beta_{i} u-\sin \beta_{i} u\right)\right], \\
\omega_{i} & =\beta_{i}^{2}(E I / \rho)^{0.5},
\end{aligned}
$$

where

$$
\begin{aligned}
& 1+\cos \beta_{i} l_{5} \cosh \beta_{i} l_{5}=0, \\
& \sigma_{i}=\frac{\cos \beta_{i} l_{5}+\cosh \beta_{i} l_{5}}{\sin \beta_{i} l_{5}+\sinh \beta_{i} l_{5}},
\end{aligned}
$$

and $i=1, \cdots \infty$.

Table 4 shows the natural frequencies and related constants for the first 4 modes of link 5. Note that the natural frequencies listed in table 4 are for link 5 without point mass at its tip.

\subsection{Manipulator dynamics}

The method used in the dynamic modelling of the system is the Lagrange-Euler formulation. In this method, the computation of the kinetic and potential energy of the system is an integral part of the formulation. As the detailed derivation of the dynamic equations is very long and tedious, only the relevant equations are presented here. For more details on the derivation, please refer to appendix A.

The full physical system comprises links 1 through 5, 2 direct drive brushless motors and a camera-LED assembly mounted on the arm. The dynamic model of the system is given by (please refer to appendix $\mathrm{A}$ )

$$
\begin{aligned}
& D(Q) \ddot{Q}+G Q+H(Q, \dot{Q})+\mu\left(\dot{\theta}_{1}, \dot{\theta}_{2}\right)=P T_{m}, \\
& Q=\left[\theta_{1} \theta_{2} q_{1} q_{2} \cdots q_{\infty}\right]^{T}, \quad T_{m}=\left[\tau_{m 1} \tau_{m 2}\right]^{T},
\end{aligned}
$$




$$
\begin{aligned}
& D(Q)=
\end{aligned}
$$

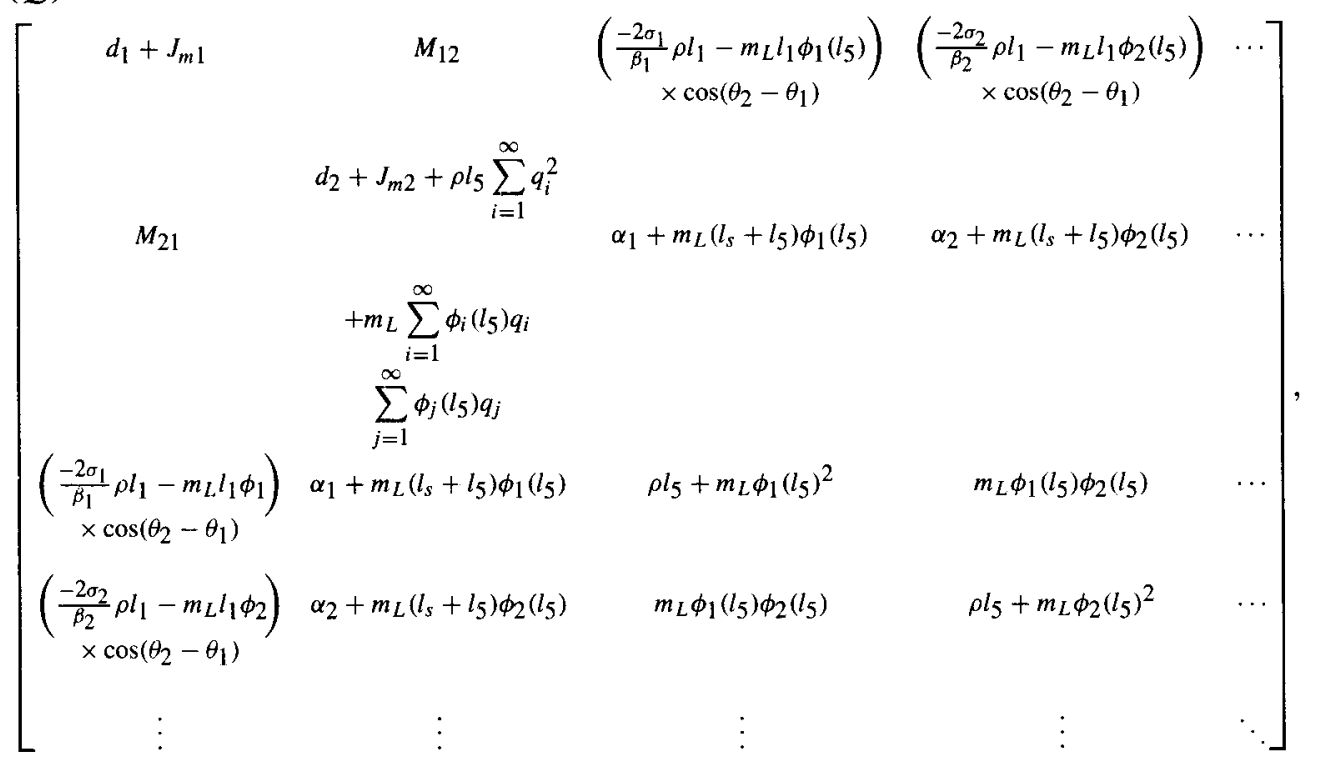

$$
\begin{array}{ccc}
G=\left[\begin{array}{ccccc}
0 & 0 & 0 & 0 & \cdots \\
0 & 0 & 0 & 0 & \cdots \\
0 & 0 & \rho l_{5} \omega_{1}^{2} & 0 & \cdots \\
0 & 0 & 0 & \rho l_{5} \omega_{2}^{2} & \cdots \\
\vdots & \vdots & \vdots & \vdots & \ddots
\end{array}\right], \\
\mu\left(\dot{\theta}_{1}, \dot{\theta}_{2}\right)=\left[\begin{array}{c}
B_{m 1} \dot{\theta}_{1}+b_{m 1} \operatorname{sgn}\left(\dot{\theta}_{1}\right) \\
B_{m 2} \dot{\theta}_{2}+b_{m 2} \operatorname{sgn}\left(\dot{\theta}_{2}\right) \\
0 \\
0 \\
\vdots
\end{array}\right], \quad P=\left[\begin{array}{cc}
1 & 0 \\
0 & 1 \\
0 & 0 \\
0 & 0 \\
\vdots & \vdots
\end{array}\right],
\end{array}
$$$$
H(Q, \dot{Q})=
$$$$
\left[\begin{array}{l}
-d_{3} \sin \left(\theta_{2}-\theta_{1}\right) \dot{\theta}_{2}^{2}+4 \rho l_{1} \dot{\theta}_{2} \sin \left(\theta_{2}-\theta_{1}\right) \sum_{i=1}^{\infty} \frac{\sigma_{i}}{\beta_{i}} \dot{q}_{i} \\
+2 m_{L} l_{1} \sin \left(\theta_{2}-\theta_{1}\right) \dot{\theta}_{2} \sum_{i=1}^{\infty} \phi_{i}\left(l_{5}\right) \dot{q}_{i} \\
+\left(2 \rho l_{1} \sum_{i=1}^{\infty} \frac{\sigma_{i}}{\beta_{i}} q_{i}+m_{L} l_{1} \sum_{i=1}^{\infty} \phi_{i}\left(l_{5}\right) q_{i}\right) \cos \left(\theta_{2}-\theta_{1}\right) \dot{\theta}_{2}^{2}
\end{array}\right],
$$ 


$$
\begin{aligned}
& {\left[\begin{array}{c}
d_{3} \sin \left(\theta_{2}-\theta_{1}\right) \dot{\theta}_{1}^{2}+2 \rho l_{5} \dot{\theta}_{2} \sum_{i=1}^{\infty} q_{i} \dot{q}_{i}+2 m_{L} \sum_{i=1}^{\infty} \phi_{i}\left(l_{5}\right) q_{i} \sum_{j=1}^{\infty} \phi_{j}\left(l_{5}\right) \dot{q}_{j} \dot{\theta}_{2} \\
-\left(2 \rho l_{1} \sum_{i=1}^{\infty} \frac{\sigma_{i}}{\beta_{i}} q_{i}+m_{L} l_{1} \sum_{i=1}^{\infty} \phi_{i}\left(l_{5}\right) q_{i}\right) \cos \left(\theta_{2}-\theta_{1}\right) \dot{\theta}_{1}^{2} \\
-\left(2 \frac{\sigma_{1}}{\beta_{1}} \rho l_{1}+m_{L} l_{1} \phi_{1}\left(l_{5}\right)\right) \sin \left(\theta_{2}-\theta_{1}\right) \dot{\theta}_{1}^{2} \\
-\left(\rho l_{5} q_{1}+m_{L}\left(\sum_{i=1}^{\infty} \phi_{i}\left(l_{5}\right) q_{i}\right) \phi_{1}\left(l_{5}\right)\right) \dot{\theta}_{2}^{2} \\
-\left(2 \frac{\sigma_{2}}{\beta_{2}} \rho l_{1}+m_{L} l_{1} \phi_{2}\left(l_{5}\right)\right) \sin \left(\theta_{2}-\theta_{1}\right) \dot{\theta}_{1}^{2} \\
-\left(\rho l_{5} q_{2}+m_{L}\left(\sum_{i=1}^{\infty} \phi_{i}\left(l_{5}\right) q_{i}\right) \phi_{2}\left(l_{5}\right)\right) \dot{\theta}_{2}^{2} \\
\vdots
\end{array}\right]} \\
& \begin{aligned}
d_{1}= & I_{1}+m_{1} l_{c 1}^{2}+I_{3}+m_{3} l_{c 3}^{2}+m_{4} l_{1}^{2}+m_{5} l_{1}^{2}+m_{c} l_{1}^{2}+m_{L} l_{1}^{2}, \\
d_{2}= & I_{2}+m_{2} l_{c 2}^{2}+I_{4}+m_{3} l_{2}^{2}+m_{4} l_{c 4}^{2}+\frac{1}{3} m_{5} l_{5}^{2}+m_{5} l_{s}^{2} \\
& \quad+m_{5} l_{s} l_{5}+m_{c} l_{c}^{2}+m_{L}\left(l_{s}+l_{5}\right)^{2} \\
d_{3}= & m_{3} l_{2} l_{c 3}-\frac{1}{2} m_{5} l_{1} l_{5}+m_{4} l_{1} l_{c 4}-m_{5} l_{s} l_{1}-m_{L} l_{1}\left(l_{s}+l_{5}\right)+m_{c} l_{1} l_{c}, \\
M_{12}= & M_{21}=d_{3} \cos \left(\theta_{2}-\theta_{1}\right)
\end{aligned} \\
& +\left[2 \rho l_{1} \sum_{i=1}^{\infty} \frac{\sigma_{i}}{\beta_{i}} q_{i}+m_{L} l_{1} \sum_{i=1}^{\infty} \phi_{i}\left(l_{5}\right) q_{i}\right] \sin \left(\theta_{2}-\theta_{1}\right), \\
& \alpha_{i}=2 \rho\left(l_{s} \frac{\sigma_{i}}{\beta_{i}}+\frac{1}{\beta_{i}^{2}}\right) \text {. }
\end{aligned}
$$

Note that the vector $T_{m}$ denotes the vector of motor torques, $J_{m_{1}}, J_{m_{2}}$ denote the rotor inertias of the motors which is given in table $1, B_{m_{1}}, B_{m_{2}}$ denote the viscous damping coefficients of the motors, and $b_{m_{1}}, b_{m_{2}}$ denote the friction coefficients of the motors. The values for all the other parameters in the dynamic model (8) may be obtained from the data in tables 2,3 and 4.

In $(8), D(Q)$ is the inertia matrix, $G(Q)$ is the stiffness matrix, $H(Q, \dot{Q})$ is a matrix containing nonlinear centrifugal and coriolis terms, and $\mu\left(\dot{\theta}_{1}, \dot{\theta}_{2}\right)$ is the friction and damping matrix. It should be noted that $\omega_{i}$ in the stiffness matrix refers to the free natural frequency of vibration of a clamped-free uniform Bernoulli-Euler beam. It does not indicate the frequency of vibration of flexible link 5. The Rayleigh's method for approximating the fundamental frequency of a continuous system is presented in $\S 4$. This method will be used to estimate the frequency of vibration of link 5 . 


\section{Control design based on reduced-order computed torque method}

In this section, a controller is designed based on a reduced-order dynamic model of the manipulator using the computer torque control design methodology. This method is referred to as the reduced-order computed torque (ROCT) method. For control of a fully rigid manipulator arm, it is only necessary to control the rigid modes of the manipulator and the computed torque method can be used effectively for this purpose. An attempt to use the same model-based control law in a manipulator with link flexibility may result in deterioration in performance because the controller has been designed to only control the rigid modes but not the flexible modes of the manipulator. The ROCT method may be used to control a flexible-link manipulator with minimal deterioration in performance, if the discarded terms in the model-based control law have only a negligible effect on the dynamics of the system. For example, friction, viscous damping or flexibility in the system may be ignored if the system is well lubricated or is fairly rigid.

The objective of this experiment is to study the deterioration in performance, if any, of the controller designed based on the ROCT method. The physical system is described by the matrix equation given by (8). Assuming link 5 is rigid, $q_{1}, q_{2}, \ldots, q_{\infty}$ and $\dot{q}_{1}, \dot{q}_{2}, \ldots, \dot{q}_{\infty}$ are set equal to zero in (8) so that the resulting equation is the dynamic model of an equivalent 'rigid' manipulator. This equation is given by

$$
\begin{gathered}
{\left[\begin{array}{c}
\tau_{m 1} \\
\tau_{m 2}
\end{array}\right]=\left[\begin{array}{cc}
d_{1}+J_{m 1} & d_{3} \cos \left(\theta_{2}-\theta_{1}\right) \\
d_{3} \cos \left(\theta_{2}-\theta_{1}\right) & d_{2}+J_{m 2}
\end{array}\right]\left[\begin{array}{l}
\ddot{\theta}_{1} \\
\ddot{\theta}_{2}
\end{array}\right]+\left[\begin{array}{c}
-d_{3} \sin \left(\theta_{2}-\theta_{1}\right) \dot{\theta}_{2}^{2} \\
d_{3} \sin \left(\theta_{2}-\theta_{1}\right) \dot{\theta}_{1}^{2}
\end{array}\right]} \\
+\left[\begin{array}{cc}
B_{m 1} & 0 \\
0 & B_{m 2}
\end{array}\right]\left[\begin{array}{c}
\dot{\theta}_{1} \\
\dot{\theta}_{2}
\end{array}\right]+\left[\begin{array}{l}
b_{m 1} \operatorname{sgn}\left(\dot{\theta}_{1}\right) \\
b_{m 2} \operatorname{sgn}\left(\dot{\theta}_{2}\right)
\end{array}\right] .
\end{gathered}
$$

Based on the reduced-order dynamic model given by (9) and ignoring the coulomb friction and viscous damping terms which are not known exactly, a control law is obtained based on the computed torque method which is given by

$$
\left[\begin{array}{c}
\tau_{m 1} \\
\tau_{m 2}
\end{array}\right]=M\left(\theta_{1}, \theta_{2}\right)\left[\begin{array}{l}
\ddot{\theta}_{1 d}+k_{p 1} e_{1}+k_{\nu 1} \dot{e}_{1}+k_{i 1} \int_{0}^{t} e_{1} \mathrm{~d} t \\
\ddot{\theta}_{2 d}+k_{p 2} e_{2}+k_{\nu 2} \dot{e}_{2}+k_{i 2} \int_{0}^{t} e_{2} \mathrm{~d} t
\end{array}\right]+h\left(\theta_{1}, \theta_{2}, \dot{\theta}_{1}, \dot{\theta}_{2}\right)
$$

where

$$
\begin{aligned}
M\left(\theta_{1}, \theta_{2}\right) & =\left[\begin{array}{cc}
d_{1}+J_{m 1} & d_{3} \cos \left(\theta_{2}-\theta_{1}\right) \\
d_{3} \cos \left(\theta_{2}-\theta_{1}\right) & d_{2}+J_{m 2}
\end{array}\right] \\
h\left(\theta_{1}, \theta_{2}, \dot{\theta}_{1}, \dot{\theta}_{2}\right) & =\left[\begin{array}{c}
-d_{3} \sin \left(\theta_{2}-\theta_{1}\right) \dot{\theta}_{2}^{2} \\
d_{3} \sin \left(\theta_{2}-\theta_{1}\right) \dot{\theta}_{1}^{2}
\end{array}\right] \\
e_{1} & =\theta_{1 d}-\theta_{1} \\
e_{2} & =\theta_{2 d}-\theta_{2}
\end{aligned}
$$

The control law given by (10) is implemented to study position and vibration control of the end effector of the manipulator. Figure 5 shows the controller based on ROCT method which has been implemented on the system. Figure 6 shows the experimental results of 


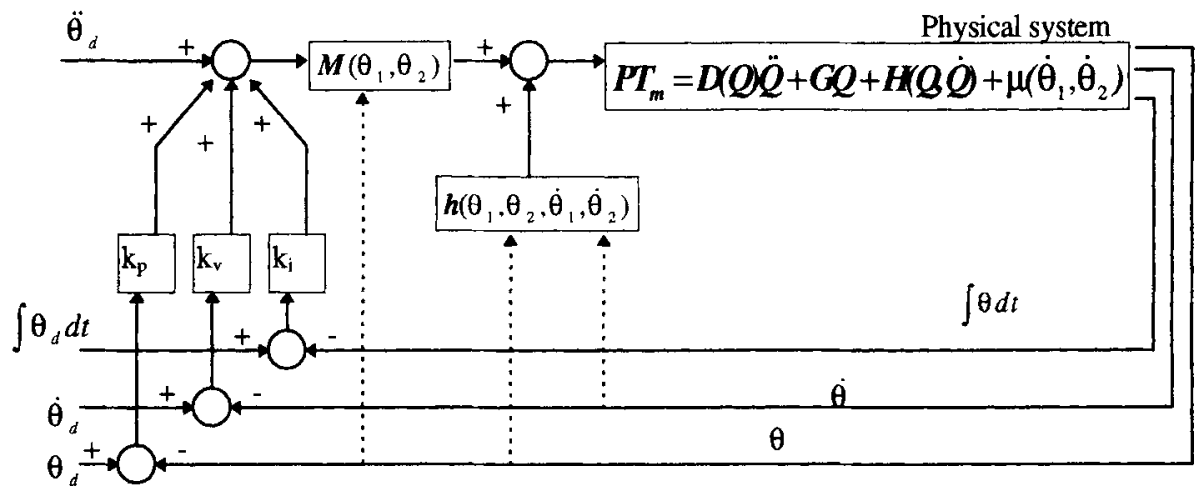

Figure 5. Controller based on reduced- order computed torque method.
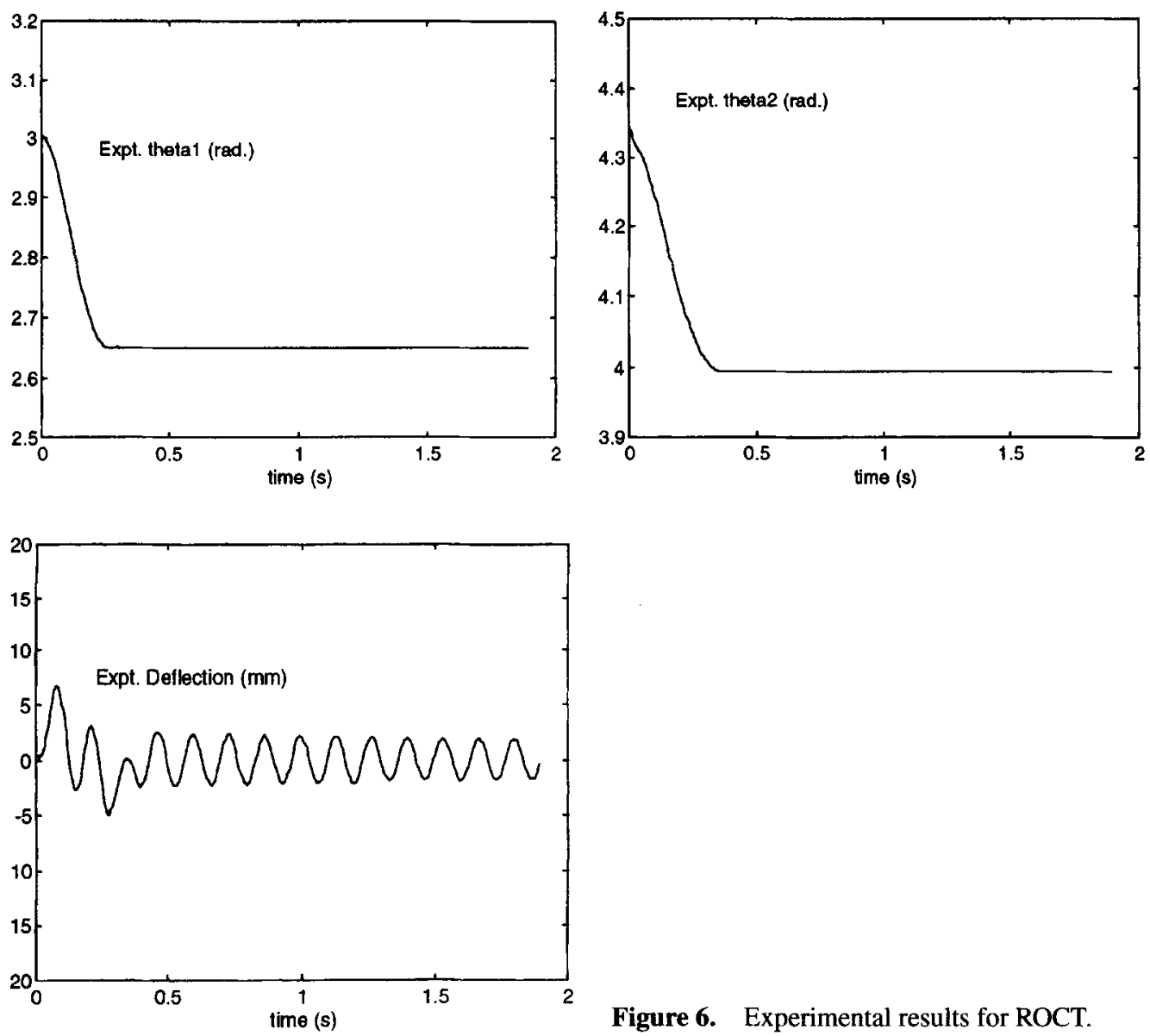

Figure 6. Experimental results for ROCT. 


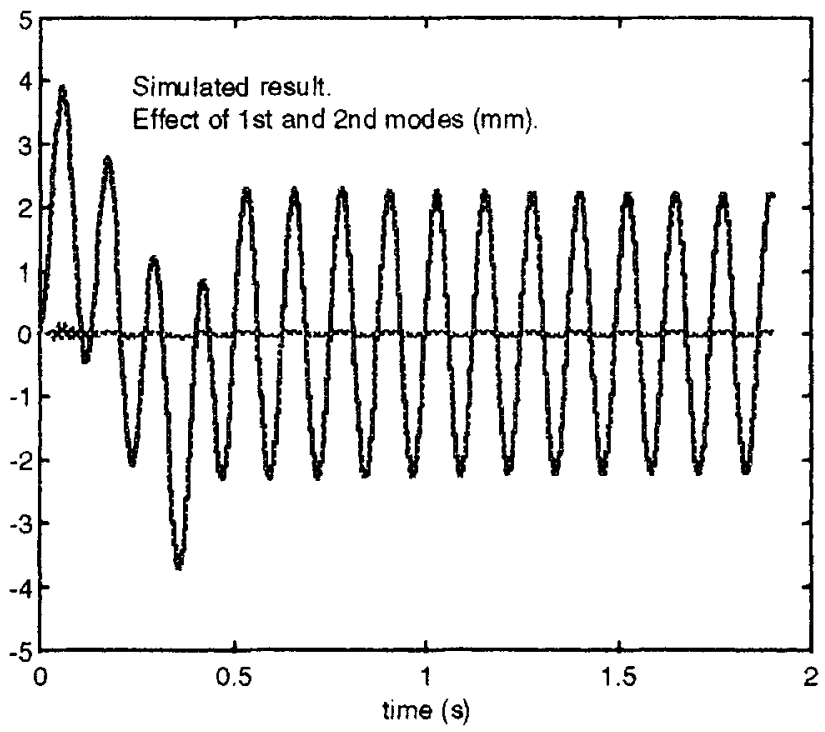

Figure 7. Relative magnitude of $\phi_{1}\left(l_{5}\right) q_{1}(t)$ and $\phi_{2}\left(l_{5}\right) q_{2}(t)$.

the ROCT method. Note that the desired state of the system is defined as $\theta_{1}=2.65 \mathrm{rad}$, $\theta_{2}=3.99 \mathrm{rad}$. The gains are set to $K p_{1}=100, K v_{1}=1$ and $K p_{2}=50, K v_{2}=4.25$.

Figure 6 shows that there is deterioration in the performance of the controller based on the ROCT method. Although the controller is able to control the rigid body modes of the manipulator, it is unable to control the vibratory modes of flexible link 5 . Thus it is necessary to use a different control law for position and vibration control of the manipulator.

Rayleigh's method for approximation of the fundamental frequency of vibration is used to estimate the first fundamental frequency of vibration. Rayleigh's quotient is given by

$$
\omega_{i}=\left\{\int_{0}^{l_{5}} E I \phi_{i}^{\prime \prime}(u)^{2} \mathrm{~d} u / \int_{0}^{l_{5}} \rho \phi_{i}(u)^{2} \mathrm{~d} u+m_{L} \phi_{i}\left(l_{5}\right)\right\}^{1 / 2}
$$

Since $\int_{0}^{l_{5}} E I \phi_{i}^{\prime \prime}(u)^{2} \mathrm{~d} u=\omega_{i}^{2} \rho l_{5}, \int_{0}^{l_{5}} \rho \phi_{i}(u)^{2} \mathrm{~d} u=\rho l_{5}$ and all the other parameters are known, the first fundamental frequency of vibration can be estimated. The parameter values were substituted into (11) and $\omega_{1}$ was found to be $8.09 \mathrm{~Hz}$. From figure 6 , it is observed that the natural frequency of vibration is about $7-8 \mathrm{~Hz}$, which is very close to $\omega_{1}$. Hence, it can be deduced that, for the experimental system, the dominant mode of vibration is mode 1 . This can also be verified by checking the relative magnitude of $\phi_{1}\left(l_{5}\right) q_{1}(t)$ and $\phi_{2}\left(l_{5}\right) q_{2}(t)$. Figure 7 shows the relative magnitude of $\phi_{1}\left(l_{5}\right) q_{1}(t)$ and $\phi_{2}\left(l_{5}\right) q_{2}(t)$ obtained from simulation. From the plots in figure 7, it is obvious that $\phi_{1}\left(l_{5}\right) q_{1}(t)$ is large compared to $\phi_{2}\left(l_{5}\right) q_{2}(t)$. The deflection at the tip of link 5 can thus be approximated by

$$
v\left(l_{5}, t\right)=\phi_{1}\left(l_{5}\right) q_{1}(t)
$$

This is because mode 1 is the dominant mode of vibration and the effects of mode 2 and other higher order modes are negligible and can be discarded. 


\section{Control design based on linear state feedback method}

The controller based on the reduced-order computed torque (ROCT) method has been presented in the previous section. In this section a control strategy based on local linearization of the nonlinear dynamic model of the physical system and linear state feedback control method is presented. Then another control strategy, referred to as the hybrid controller. which combines the positive characteristics of both the ROCT controller and state feedback control law is presented.

Recall that the matrix representation that describes the dynamics of the experimental system is given by

$$
D(Q) \ddot{Q}+G Q+H(Q, \dot{Q})+\mu\left(\theta_{1}, \dot{\theta}_{2}\right)=P T_{m} .
$$

It has been deduced in $\S 4$ that the dominant mode of vibration for the experimental system is mode 1 . The effect of mode 2 and other higher order modes is negligible so that they may be ignored. In general, for other systems, more than one mode may be required to give a reasonable approximation for vibration. When more than one mode is used, not all states are measurable based on measurements from the encoders and the PSD. Therefore an observer must be constructed so as to estimate the unmeasurable states. In such a case, the design of the controller becomes more complex because if the system is of $n$th order, the observer is also of $n$th order for a full order state observer. The resulting closed loop system becomes of order $2 n$; that is the complexity and size of the problem is doubled. Furthermore, some deterioration in performance may be observed if the dynamic response of the observer is not good. If only a 1-mode approximation is used, it eliminates the need for an observer which is clear from (12). For the experimental system, a controller based on this model is implemented and satisfactory results are obtained.

Define the state vector to be $x=\left[\theta_{1} \theta_{2} q_{1} \dot{\theta}_{1} \dot{\theta}_{2} \dot{q}\right]^{T}$ and the equilibrium state to be $x_{0}=$ $\left[\theta_{1}^{0} \theta_{2}^{0} 00000\right]^{T}$, where $\theta_{1}^{0}$ and $\theta_{2}^{0}$ are the desired joint angles. The linearized equations of the experimental system with 1 -mode approximation for the flexibilty is defined as follows.

$$
\begin{aligned}
\delta \dot{x} & =A \delta x+B \delta u, \\
\delta x & =\left[\delta \theta_{1} \delta \theta_{2} \delta q_{1} \delta \dot{\theta}_{1} \delta \dot{\theta}_{2} \delta \dot{q}_{1}\right]^{T}, \\
A & =\left(\begin{array}{cc}
0 & I \\
-\left.\tilde{D}(Q)\right|_{0} ^{-1} \tilde{G} & 0
\end{array}\right), B=\left(\begin{array}{c}
0 \tilde{P} \\
\left.\tilde{D}(Q)\right|_{0} ^{-1} \tilde{P}
\end{array}\right),
\end{aligned}
$$

0 is a $3 \times 3$ null matrix, $I$ is a $3 \times 3$ identity matrix,

$$
\begin{array}{cl}
\delta u & =\delta T_{m}=\left[\tau_{m 1} \tau_{m 2}\right]^{T}, \quad \tilde{P}=\left[\begin{array}{lll}
1 & 0 & 0 \\
0 & 1 & 0
\end{array}\right]^{T}, \\
\tilde{G} & =\left[\begin{array}{ccc}
0 & 0 & 0 \\
0 & 0 & 0 \\
0 & 0 & \rho l_{5} \omega_{1}^{2}
\end{array}\right]
\end{array}
$$


and

$$
\left.\begin{array}{ccc}
\left.\tilde{D}(Q)\right|_{0}= & \\
d_{1}+J_{m 1} & d_{3} \cos \left(\theta_{2}^{0}-\theta_{1}^{0}\right) & \left(\frac{-2 \sigma_{1}}{\beta_{1}} \rho l_{1}-m_{L} l_{1} \phi_{1}\left(l_{5}\right)\right) \\
d_{3} \cos \left(\theta_{2}^{0}-\theta_{1}^{0}\right) & d_{2}+J_{m 2} & \times\left(\theta_{2}^{0}-\theta_{1}^{0}\right) \\
\left(\frac{-2 \sigma_{1}}{\beta_{1}} \rho l_{1}-m_{L} l_{1} \phi_{1}\left(l_{5}\right)\right) & \alpha_{1}+m_{L}\left(l_{s}+l_{5}\right) \phi_{1}\left(l_{5}\right) & \rho l_{5}+m_{L} \phi_{1}\left(l_{5}\right)^{2} \\
\times \cos \left(\theta_{2}^{0}-\theta_{1}^{0}\right) &
\end{array}\right) .
$$

The state feedback controller for the linearized system is implemented and experimental results are obtained. The control law is given by

$$
\left[\begin{array}{c}
\tau_{m 1} \\
\tau_{m 2}
\end{array}\right]=-K\left[\begin{array}{c}
\theta_{1}-\theta_{1}^{0} \\
\theta_{2}-\theta_{2}^{0} \\
q_{1} \\
\dot{\theta}_{1} \\
\dot{\theta}_{2} \\
\dot{q}_{1}
\end{array}\right] .
$$

The feedback gain matrix is chosen to be

$$
K=\left[\begin{array}{cccccc}
34.63 & -0.58 & 16.50 & 6.33 & 0.01 & 1.01 \\
0.29 & 7.07 & -7.56 & 0.14 & 1.88 & -0.64
\end{array}\right]
$$

which gives the following closed loop eigenvalues for the system as

$$
e=\left[\begin{array}{c}
-0.92 \pm 53.07 i \\
-6.30 \pm 5.43 i \\
-4.00 \pm 3.75 i
\end{array}\right]
$$

Figure 8 shows the experimental results of this controller, in which the desired state of the system is defined as

$$
\theta_{1}^{0}=2.65 \mathrm{rad}, \quad \theta_{2}^{0}=3.99 \mathrm{rad}, \quad q_{1}^{0}=0, \quad \dot{\theta}_{1}^{0}=0, \quad \dot{\theta}_{1}^{0}=0, \quad \dot{q}_{1}^{0}=0 .
$$

Comparing figures 8 and 6, it is clear that slower response is observed for the rigid body modes using the state feedback control based on the linearized state space model in comparison to that obtained by the ROCT method. However, vibration at the tip of link 5 is suppressed after a very short time. The experimental results show the effect of the state feedback controller designed based on the linearized system obtained by a local linearization of the nonlinear dynamic model, given in (13), about an equilibrium state. When nonlinearities in the system are not severe, local linearization may be used as an approximation to the nonlinear model. However, such manipulator control is not well suited for the manipulator making a large movement. An alternative is to use gain scheduling, in which the controller gain matrix and the equilibrium state of the manipulator change with the manipulator as it moves. 

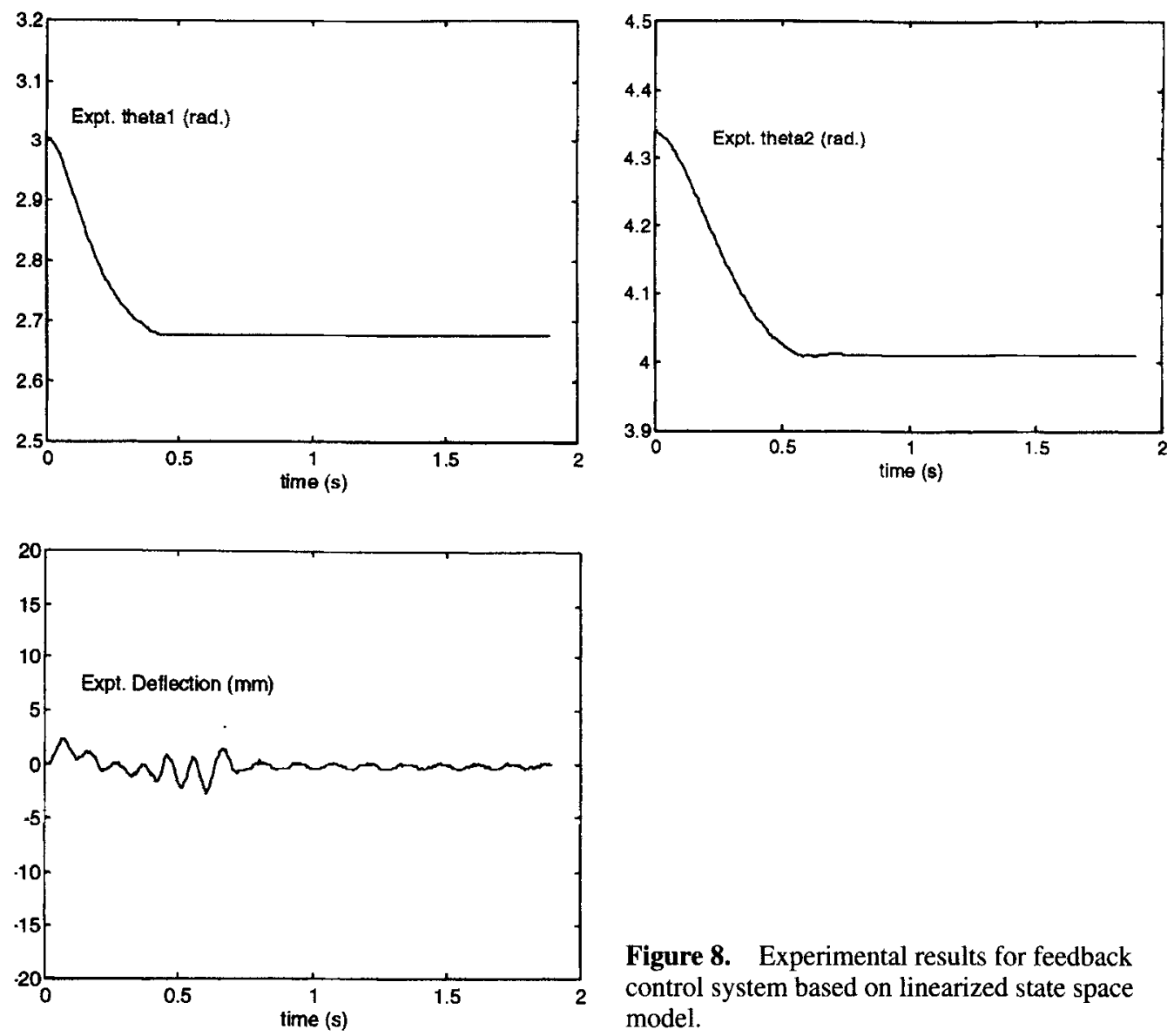

Figure 8. Experimental results for feedback control system based on linearized state space model.

A hybrid controller is proposed as another alternative to control the flexible-link manipulator. The hybrid controller comprises the controller based on the reduced-order computed torque (ROCT) method for the initial large movement of the manipulator, i.e. from the initial state to a state sufficiently close to the desired state. When the manipulator is close to the desired state, the controller based on the ROCT method is switched to the linear state feedback controller. A switching rule is used for such purpose. Figure 9 shows the experimental results for the hybrid controller. The initial state and desired state of the system is set identical to that defined in ROCT and state feedback control. The same gains are also used. The switching rule is to switch from ROCT controller to state feedback controller when both $\theta_{1}$ and $\theta_{2}$ are within $10^{\circ}$ from the desired angles.

The experimental results show that there is significant improvement in the performance of the controller in terms of speed of response of the angular positions and vibration suppression at the tip of the manipulator. The use of the ROCT controller for the initial large movement of the manipulator enables fast response for the angular position. When the manipulator moves to a state sufficiently close to the desired state, where the local linearization of the nonlinear system is valid, the controller is switched to state feedback control. This controller suppresses the vibration at the tip of the manipulator figure 10 compares the experimental results of the 3 controllers, which reiterates the above discussion. 

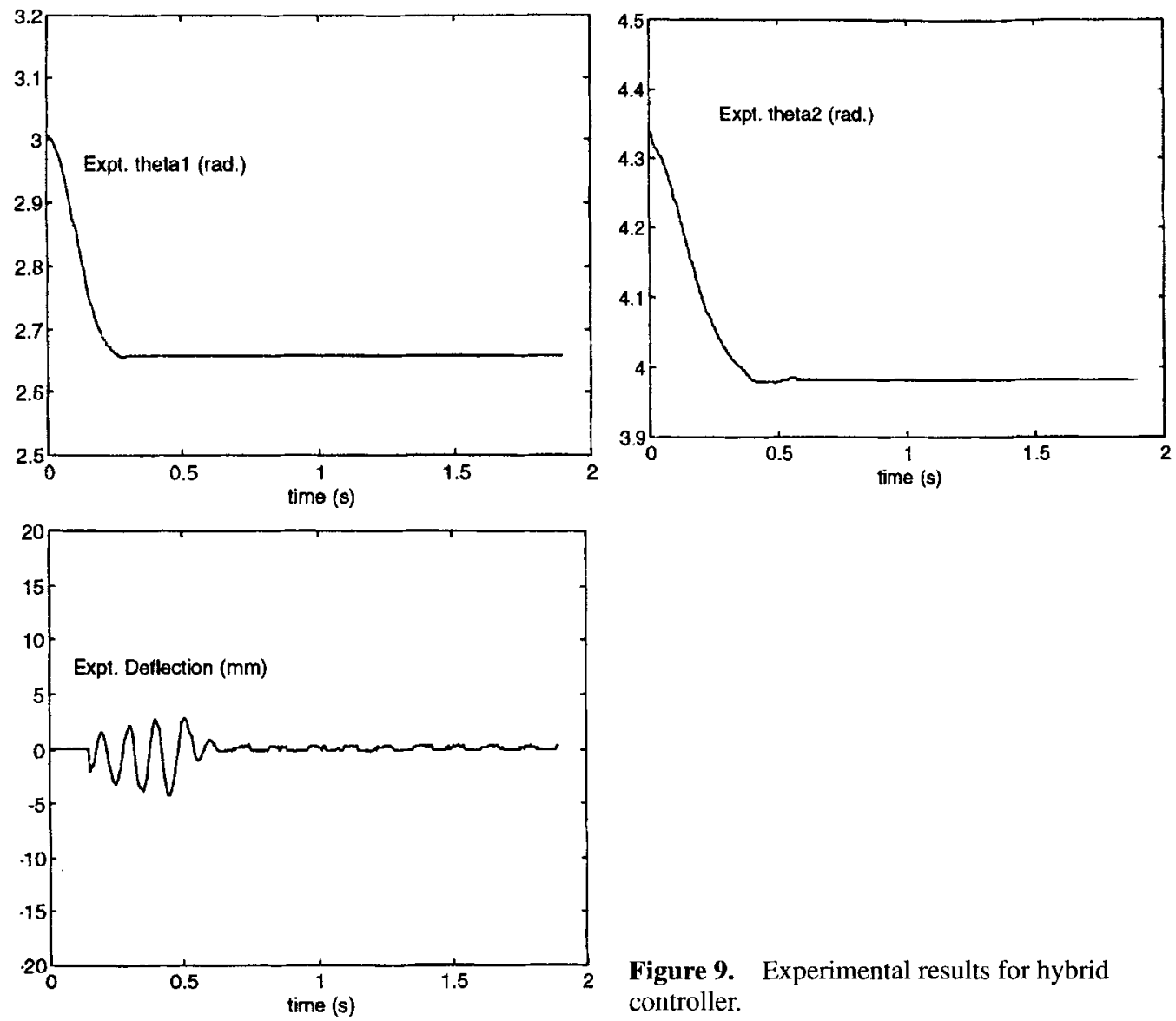

Figure 9. Experimental results for hybrid controller.

\section{Conclusion}

In this paper, experimental results on the position and vibration control of the end-effector of a 2-DOF manipulator with a flexible forearm have been presented. The dynamic model is obtained using the Lagrange Euler formulation and by modelling the vibration using the assumed-mode method. Control strategies are implemented to control the manipulator. The first control strategy uses the computed torque method based on a reduced-order dynamic model of the manipulator obtained under the assumption that all the links are rigid. This method has been referred to as the reduced-order computed torque (ROCT) method. Experimental results show that the ROCT controller is not suitable for position and vibration control. However, the ROCT method is good in controlling the rigid modes (angular positions) of the manipulator. The second control strategy is a state feedback control law designed based on a local linearization of the nonlinear dynamic model about an equilibrium state. Experimental results show that the performance of the state feedback control law is good and the vibration at the end-effector of the manipulator is damped out effectively. However this controller is valid only for small movement of the manipulator about the equilibrium state because linearization is done locally. The third control strategy combines the positive characteristics of each of the two controllers described above. This controller, referred to as the hybrid controller, uses the ROCT method for the initial large 

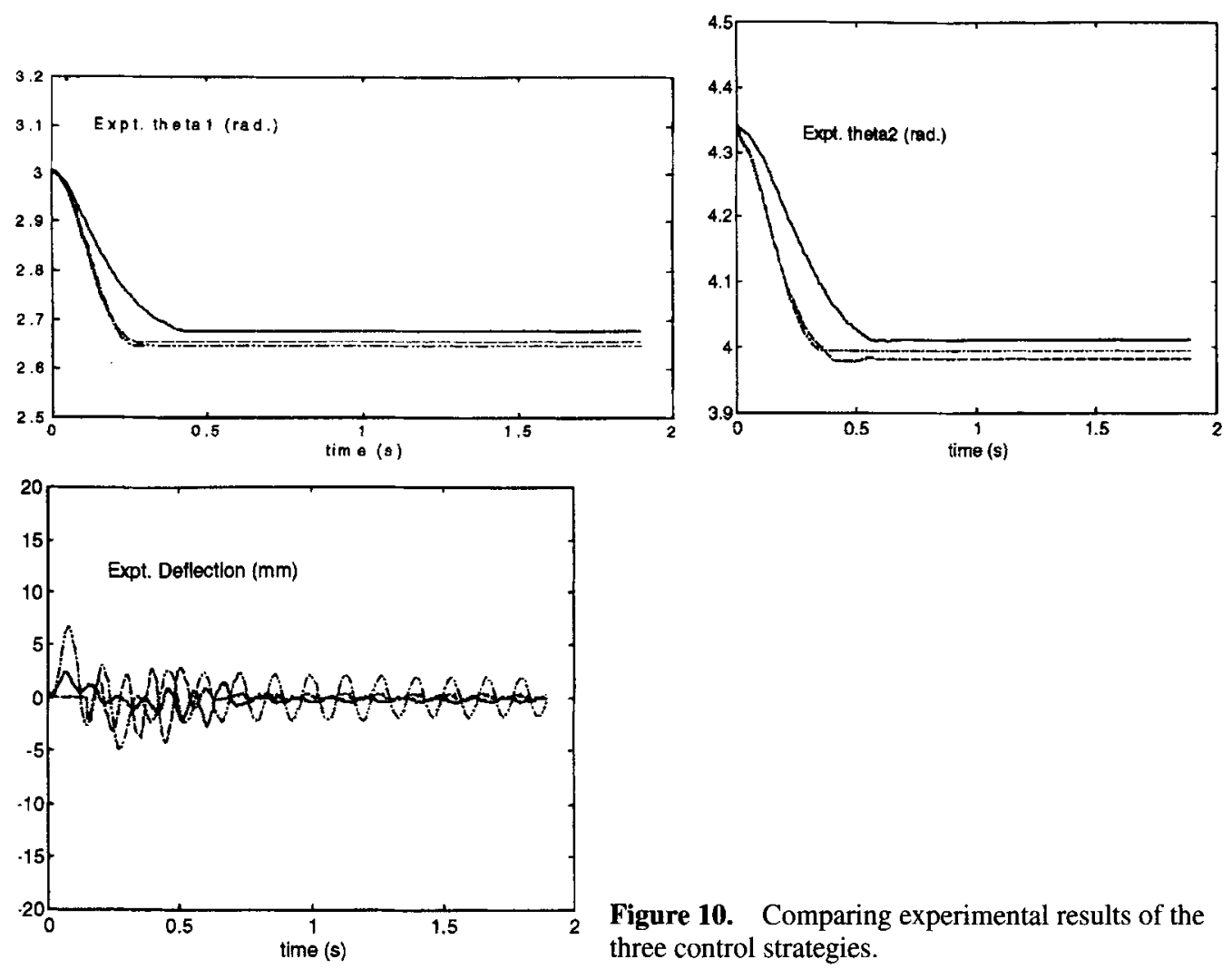

Figure 10. Comparing experimental results of the three control strategies.

movement of the manipulator. Based on a switching rule, the controller is switched to the state feedback controller when the manipulator is sufficiently close to the equilibrium state. Experimental results show good speed of response and effective vibration suppression at the end-effector of the manipulator. We mention that a one-mode approximation of the flexible modes was used in our experiments in control of the 2-DOF manipulator. If more than one mode of vibration is dominant in the system, then for vibration control one must design a state estimator for estimating the flexible modes of the manipulator based on the deflection measurements obtained using the PSD.

\section{Appendix A.}

In this section we provide the detailed derivation of the equations of motion for the 2DOF manipulator with a flexible forearm. The full dynamic model of the system includes modelling of manipulator, modelling of point masses and modelling of the motors. The following assumptions are made on the flexible link 5 (see figure 1), in the derivation of the dynamic equations of the manipulator:

(1) Link 5 is linearly elastic and vibrates horizontally.

(2) Shear deformation and rotary inertia of link 5 are negligible.

(3) Deflection of link 5 is small and change in length of the beam is negligible. 
(4) Since the manipulator moves horizontally, the only potential energy is the elastic potential energy of link 5 .

Lagrange's equation is given by

$$
\frac{d}{d t}\left(\frac{\partial T}{\partial \dot{z}_{k}}\right)-\frac{\partial T}{\partial z_{k}}+\frac{\partial U}{\partial z_{k}}=F_{k}, \quad k=1,2,3, \ldots, \infty,
$$

where $z_{k}$ is the generalized coordinate and $F_{k}$ is the generalized force. The velocity of an arbitrary point $R$ on link 5 (see figure 1 ) is given by

$$
\tilde{v}_{R}=\left[\begin{array}{cc}
-l_{1} \sin \theta_{1} & \left(l_{s}+u\right) \sin \theta_{2} \\
l_{1} \cos \theta_{1} & -\left(l_{s}+u\right) \cos \theta_{2}
\end{array}\right]\left[\begin{array}{c}
\dot{\theta}_{1} \\
\dot{\theta}_{2}
\end{array}\right]+\left[\begin{array}{c}
\sin \theta_{2}(\partial v / \partial t)+v \dot{\theta}_{2} \cos \theta_{2} \\
-\cos \theta_{2}(\partial v / \partial t)+v \dot{\theta}_{2} \sin \theta_{2}
\end{array}\right] \text {. }
$$

The kinetic energy of links 1 to 5 are given by

$$
\begin{aligned}
T_{1} & =\frac{1}{2} I_{1} \dot{\theta}_{1}^{2}+\frac{1}{2} m_{1} l_{c 1}^{2} \dot{\theta}_{1}^{2}, \\
T_{2} & =\frac{1}{2} I_{2} \dot{\theta}_{2}^{2}+\frac{1}{2} m_{2} l_{c 2}^{2} \dot{\theta}_{2}^{2} \\
T_{3} & =\frac{1}{2} I_{3} \dot{\theta}_{1}^{2}+\frac{1}{2} m_{3}\left[l_{c 3}^{2} \dot{\theta}_{1}^{2}+l_{2}^{2} \dot{\theta}_{2}^{2}+2 l_{2} l_{c 3} \dot{\theta}_{1} \dot{\theta}_{2} \cos \left(\theta_{2}-\theta_{1}\right)\right] \\
T_{4} & =\frac{1}{2} I_{4} \dot{\theta}_{2}^{2}+\frac{1}{2} m_{4}\left[l_{1}^{2} \dot{\theta}_{1}^{2}+l_{c 4}^{2} \dot{\theta}_{2}^{2}+2 l_{1} l_{c 4} \dot{\theta}_{1} \dot{\theta}_{2} \cos \left(\theta_{2}-\theta_{1}\right)\right] \\
T_{5}= & \frac{1}{2} \int \tilde{v}_{R x}^{2}+\tilde{v}_{R y}^{2} d m \\
= & \frac{1}{2} \rho \int_{0}^{l_{5}}\left(-l_{1} \dot{\theta}_{1} \sin \theta_{1}+\left(l_{s}+u\right) \dot{\theta} \sin \theta_{2}+\sin \theta_{2} \frac{\partial v}{\partial t}+v \dot{\theta}_{2} \cos \theta_{2}\right)^{2} \\
& \quad+\left(l_{1} \dot{\theta}_{1} \cos \theta_{1}-\left(l_{s}+u\right) \dot{\theta}_{2} \cos \theta_{2}-\cos \theta_{2} \frac{\partial v}{\partial t}+v \dot{\theta}_{2} \sin \theta_{2}\right)^{2} \mathrm{~d} u
\end{aligned}
$$

Since link 5 is a uniform beam,

$$
\begin{aligned}
m_{5} & =\rho l_{5}, \\
I_{b} & =\int_{0}^{l_{5}} u^{2} \mathrm{~d} m=\int_{0}^{l_{5}} u^{2} \rho \mathrm{d} u=\frac{1}{3} m_{5} l_{5}^{2} .
\end{aligned}
$$

Moreover, $v(u, t)=\sum_{i=1}^{\infty} \phi_{i}(u) q_{i}(t)$, therefore by expansion and simplification we get

$$
\begin{aligned}
T_{5}=\frac{1}{2} m_{5} l_{1}^{2} \dot{\theta}_{1}^{2}+\frac{1}{2}\left[I_{b}+m_{5} l_{s}^{2}+m_{5} l_{s} l_{5}\right] \dot{\theta}_{2}^{2} \\
-\frac{1}{2} m_{5}\left[l_{1} l_{5}+2 l_{1} l_{s}\right] \dot{\theta}_{1} \dot{\theta}_{2} \cos \left(\theta_{2}-\theta_{1}\right) \\
+\frac{1}{2} \rho \int_{0}^{l_{5}}\left[\sum_{i=1}^{\infty} \phi_{i}(u) \dot{q}_{i}(t) \sum_{j=1}^{\infty} \phi_{j}(u) \dot{q}_{j}(t)\right] \\
+\left[\sum_{i=1}^{\infty} \phi_{i}(u) q_{i}(t) \sum_{j=1}^{\infty} \phi_{j}(u) q_{j}(t)\right] \dot{\theta}_{2}^{2} \\
+2\left[-l_{1} \dot{\theta}_{1} \cos \left(\theta_{2}-\theta_{1}\right) \sum_{i=1}^{\infty} \phi_{i}(u) \dot{q}_{i}(t)\right.
\end{aligned}
$$




$$
\begin{gathered}
\left.+l_{1} \dot{\theta}_{1} \dot{\theta}_{2} \sin \left(\theta_{2}-\theta_{1}\right) \sum_{i=1}^{\infty} \phi_{i}(u) q_{i}(t)\right] \\
+2\left[\left(l_{s}+u\right) \dot{\theta}_{2} \sum_{i=1}^{\infty} \phi_{i}(u) \dot{q}_{i}(t)\right] \mathrm{d} u .
\end{gathered}
$$

In the experiment, a camera-LED assembly is mounted on the manipulator. The effect of the LED assembly, mounted at the tip of flexible link 5, is to decrease the natural frequency of vibration of link 5. For modelling accuracy, the camera and LED are modelled as point masses. The following kinematic relationships are used in the derivation.

$$
\begin{aligned}
{\left[\begin{array}{l}
x_{c} \\
y_{c}
\end{array}\right]=\left[\begin{array}{l}
l_{1} \cos \theta_{1}+l_{c} \cos \theta_{2} \\
l_{1} \sin \theta_{1}+l_{c} \sin \theta_{2}
\end{array}\right], } \\
{\left[\begin{array}{l}
\dot{x}_{c} \\
\dot{y}_{c}
\end{array}\right]=\left[\begin{array}{c}
-\dot{\theta}_{1} l_{1} \sin \theta_{1} \dot{\theta}_{2} l_{c} \sin \theta_{2} \\
\dot{\theta}_{1} l_{1} \cos \theta_{1}+\dot{\theta}_{2} l_{c} \cos \theta_{2}
\end{array}\right], } \\
{\left[\begin{array}{l}
x_{e} \\
y_{e}
\end{array}\right]=\left[\begin{array}{l}
l_{1} \cos \theta_{1}-\left(l_{s}+l_{5}\right) \cos \theta_{2}+v\left(l_{5}\right) \sin \theta_{2} \\
l_{1} \sin \theta_{1}-\left(l_{s}+l_{5}\right) \sin \theta_{2}-v\left(l_{5}\right) \cos \theta_{2}
\end{array}\right], } \\
{\left[\begin{array}{l}
\dot{x}_{e} \\
\dot{y}_{e}
\end{array}\right]=\left[\begin{array}{l}
-\dot{\theta}_{1} l_{1} \sin \theta_{1}+\dot{\theta}_{2}\left(l_{s}+l_{5}\right) \sin \theta_{2}+v\left(l_{5}\right) \dot{\theta}_{2} \cos \theta_{2}+\dot{v}\left(l_{5}\right) \sin \theta_{2} \\
\dot{\theta}_{1} l_{1} \cos \theta_{1}-\dot{\theta}_{2}\left(l_{s}+l_{5}\right) \cos \theta_{2}+v\left(l_{5}\right) \dot{\theta}_{2} \sin \theta_{2}-\dot{v}\left(l_{5}\right) \cos \theta_{2}
\end{array}\right] . }
\end{aligned}
$$

Note that $\left(x_{c}, y_{c}\right)$ refers to the location of the camera and $\left(x_{e}, y_{e}\right)$ refers to the location of the LED, which is at the tip of link 5 (see figure 1). The kinetic energy of the sensor camera is given by

$$
\begin{aligned}
T_{c} & =\frac{1}{2} m_{c}\left(\dot{x}_{c}^{2}+\dot{y}_{c}^{2}\right) \\
& =\frac{1}{2} m_{c}\left[l_{1}^{2} \dot{\theta}_{1}^{2}+l_{c}^{2} \dot{\theta}_{2}^{2}+2 l_{1} l_{c} \dot{\theta}_{1} \dot{\theta}_{2} \cos \left(\theta_{2}-\theta_{1}\right)\right] .
\end{aligned}
$$

The kinetic energy of the LED is given by

$$
T_{\mathrm{LED}}=\frac{1}{2} m_{L}\left(\dot{x}_{e}^{2}+\dot{y}_{e}^{2}\right) .
$$

This can be simplified as

$$
\begin{aligned}
T_{\mathrm{LED}}=\frac{1}{2} m_{L}[ & l_{1}^{2} \dot{\theta}_{1}^{2}+\left(l_{s}+l_{5}\right)^{2} \dot{\theta}_{2}^{2}+\dot{\theta}_{2}^{2} \sum_{i=1}^{\infty} \phi_{i}\left(l_{5}\right) q_{i}(t) \\
& \left.\times \sum_{j=1}^{\infty} \phi_{j}\left(l_{5}\right) q_{j}(t)+\sum_{i=1}^{\infty} \phi_{i}\left(l_{5}\right) \dot{q}_{i}(t) \sum_{j=1}^{\infty} \phi_{j}\left(l_{5}\right) \dot{q}_{j}(t)\right] \\
& -m_{L} l_{1}\left(l_{s}+l_{5}\right) \dot{\theta}_{1} \dot{\theta}_{2} \cos \left(\theta_{2}-\theta_{1}\right)+m_{L} \\
& \times\left[l_{1} \sin \left(\theta_{2}-\theta_{1}\right) \dot{\theta}_{1} \dot{\theta}_{2} \sum_{i=1}^{\infty} \phi_{i}\left(l_{5}\right) q_{i}(t)\right. \\
& \left.+\left(l_{s}+l_{5}\right) \dot{\theta}_{2} \sum_{i=1}^{\infty} \phi_{i}\left(l_{5}\right) \dot{q}_{i}(t)\right] \\
& +m_{L}\left[-l_{1} \cos \left(\theta_{2}-\theta_{1}\right) \dot{\theta}_{1} \sum_{i=1}^{\infty} \phi_{i}\left(l_{5}\right) \dot{q}_{i}(t)\right]
\end{aligned}
$$


From (A2)-(A8), the total kinetic energy of the system is given by

$$
T=\sum_{i=1}^{5} T_{i}+T_{c}+T_{\mathrm{LED}}
$$

The eigenfunctions satisfy two orthogonality conditions (Hastings \& Book 1986). The orthogonality conditions given by Hastings \& Book (1986) are used to simplify the above expression for kinetic energy of the system. The orthogonality conditions are

$$
\begin{aligned}
\int_{0}^{l_{5}} \rho \phi_{i} \phi_{j} \mathrm{~d} u & = \begin{cases}0, & \text { if } i \neq j, \\
\rho l_{5}, & \text { if } i=j,\end{cases} \\
\int_{0}^{l_{5}} E I \phi_{i}^{\prime \prime} \phi_{j}^{\prime \prime} \mathrm{d} u & = \begin{cases}0, & \text { if } i \neq j, \\
\omega_{i}^{2} \rho l_{5}, & \text { if } i=j .\end{cases}
\end{aligned}
$$

where $\phi_{i}(u)$ is the assumed-mode defined by (4). Let

$$
\begin{aligned}
& d_{1}=I_{1}+m_{1} l_{c 1}^{2}+I_{3}+m_{3} l_{c 3}^{2}+m_{4} l_{1}^{2}+m_{5} l_{1}^{2}+m_{c} l_{1}^{2}+m_{L} l_{1}^{2} \\
& d_{2}=I_{2}+m_{2} l_{c 2}^{2}+I_{4}+m_{3} l_{2}^{2}+m_{4} l_{c 4}^{2}+\frac{1}{3} m_{5} l_{5}^{2}+m_{5} l_{s}^{2}+m_{5} l_{s} l_{5} \\
& \quad+m_{c} l_{c}^{2}+m_{L}\left(l_{s}+l_{5}\right)^{2} \\
& d_{3}=m_{3} l_{2} l_{c 3}-\frac{1}{2} m_{5} l_{1} l_{5}+m_{4} l_{1} l_{c 4}-m_{5} l_{s} l_{1}-m_{L} l_{1}\left(l_{s}+l_{5}\right)+m_{c} l_{1} l_{c} .
\end{aligned}
$$

It can be shown that

$$
\int_{0}^{l_{5}} u \phi_{i}(u) \mathrm{d} u=2 / \beta_{i}^{2},
$$

and

$$
\begin{aligned}
\int_{0}^{l_{5}} \phi_{i}(u) \mathrm{d} u & =\int_{0}^{l_{5}} \cosh \beta_{i} u-\cos \beta_{i} u-\sigma_{i}\left(\sinh \beta_{i} u-\sin \beta_{i} u\right) \mathrm{d} u \\
& =2 \sigma_{i} / \beta_{i} . \\
\therefore \int_{0}^{l_{5}}\left(l_{s}+u\right) \phi_{i}(u) \mathrm{d} u & =2\left(l_{s} \frac{\sigma_{i}}{\beta_{i}}+\frac{1}{\beta_{i}^{2}}\right)=\frac{\alpha_{i}}{\rho}, \quad \text { where } \alpha_{i}=2 \rho\left(l_{s} \frac{\sigma_{i}}{\beta_{i}}+\frac{1}{\beta_{i}^{2}}\right) .
\end{aligned}
$$

Using the orthogonality conditions and the relations mentioned above, the total kinetic energy of the system can be simplified as

$$
\begin{aligned}
T=\frac{1}{2} & d_{1} \dot{\theta}_{1}^{2}+\frac{1}{2} d_{2} \dot{\theta}_{2}^{2}+d_{3} \dot{\theta}_{1} \dot{\theta}_{2} \cos \left(\theta_{2}-\theta_{1}\right)+\frac{1}{2} \rho l_{5} \sum_{i=1}^{\infty} \dot{q}_{i}^{2}(t) \\
& +\frac{1}{2} \rho l_{5} \dot{\theta}_{2}^{2} \sum_{i=1}^{\infty} q_{i}^{2}(t)-2 \rho l_{1} \dot{\theta}_{1} \cos \left(\theta_{2}-\theta_{1}\right) \sum_{i=1}^{\infty}\left(\dot{q}_{i}(t) \frac{\sigma_{i}}{\beta_{i}}\right) \\
& +2 \rho l_{1} \dot{\theta}_{1} \dot{\theta}_{2} \sin \left(\theta_{2}-\theta_{1}\right) \sum_{i=1}^{\infty}\left(q_{i}(t) \frac{\sigma_{i}}{\beta_{i}}\right)+\dot{\theta}_{2} \sum_{i=1}^{\infty}\left(\dot{q}_{i}(t) \alpha_{i}\right)+\frac{1}{2} m_{L} \\
& \times\left[\dot{\theta}_{2}^{2} \sum_{i=1}^{\infty} \phi_{i}\left(l_{5}\right) q_{i}(t) \sum_{j=1}^{\infty} \phi_{j}\left(l_{5}\right) q_{j}(t)+\sum_{i=1}^{\infty} \phi_{i}\left(l_{5}\right) \dot{q}_{i}(t) \sum_{j=1}^{\infty} \phi_{j}\left(l_{5}\right) \dot{q}_{j}(t)\right]
\end{aligned}
$$




$$
\begin{aligned}
& +m_{L}\left[l_{1} \sin \left(\theta_{2}-\theta_{1}\right) \dot{\theta}_{1} \dot{\theta}_{2} \sum_{i=1}^{\infty} \phi_{i}\left(l_{5}\right) q_{i}(t)+\left(l_{s}+l_{5}\right) \dot{\theta}_{2} \sum_{i=1}^{\infty} \phi_{i}\left(l_{5}\right) \dot{q}_{i}(t)\right] \\
& +m_{L}\left[-l_{1} \cos \left(\theta_{2}-\theta_{1}\right) \dot{\theta}_{1} \sum_{i=1}^{\infty} \phi_{i}\left(l_{5}\right) \dot{q}_{i}(t)\right]
\end{aligned}
$$

Since the manipulator moves in a horizontal plane, the total potential energy consists of only the elastic potential energy of link 5 . The potential energy of the system is given by

$$
\begin{aligned}
& U=\frac{1}{2} \int_{0}^{l_{5}} E I\left(\frac{\partial^{2} v}{\partial u^{2}}\right)^{2} \mathrm{~d} u \\
& U=\frac{1}{2} E I \int_{0}^{l_{5}}\left[\sum_{i=1}^{\infty} \phi_{i}^{\prime \prime}(u) q_{i}(t) \sum_{j=1}^{\infty} \phi_{j}^{\prime \prime}(u) q_{j}(t)\right] \mathrm{d} u \\
& U=\frac{1}{2} \rho l_{5} \sum_{i=1}^{\infty} \omega_{i}^{2} q_{i}^{2}(t) .
\end{aligned}
$$

Using the kinetic energy and potential energy expressions (A9) and (A10), the Lagrange equation given by (Al) can now be used to find the equations of motion of the manipulator.

For generalized coordinate, $z_{1}=\theta_{1}$

$$
\begin{aligned}
& d_{1} \ddot{\theta}_{1}+d_{3} \cos \left(\theta_{2}-\theta_{1}\right) \ddot{\theta}_{2}-d_{3} \sin \left(\theta_{2}-\theta_{1}\right) \dot{\theta}_{2}^{2} \\
& -2 \rho l_{1} \cos \left(\theta_{2}-\theta_{1}\right) \sum_{i=1}^{\infty} \ddot{q}_{i}(t) \frac{\sigma_{i}}{\beta_{i}}+4 \rho l_{1} \dot{\theta}_{2} \sin \left(\theta_{2}-\theta_{1}\right) \sum_{i=1}^{\infty} \dot{q}_{i}(t) \frac{\sigma_{i}}{\beta_{i}} \\
& +2 \rho l_{1}\left[\ddot{\theta}_{2} \sin \left(\theta_{2}-\theta_{1}\right)+\dot{\theta}_{2}^{2} \cos \left(\theta_{2}-\theta_{1}\right)\right] \sum_{i=1}^{\infty} q_{i}(t) \frac{\sigma_{i}}{\beta_{i}} \\
& +m_{L} l_{1} \sin \left(\theta_{2}-\theta_{1}\right) \sum_{i=1}^{\infty} \phi_{i}\left(l_{5}\right) q_{i} \theta_{2}+2 m_{L} l_{1} \sin \left(\theta_{2}-\theta_{1}\right) \dot{\theta}_{2} \sum_{i=1}^{\infty} \phi_{i}\left(l_{5}\right) \dot{q}_{i} \\
& +m_{L} l_{1} \cos \left(\theta_{2}-\theta_{1}\right) \sum_{i=1}^{\infty} \phi_{i}\left(l_{5}\right) q_{i} \dot{\theta}_{2}^{2}-m_{L} l_{1} \cos \left(\theta_{2}-\theta_{1}\right) \sum_{i=1}^{\infty} \phi_{i}\left(l_{5}\right) \ddot{q}_{i}=\tau_{1} .
\end{aligned}
$$

For generalized coordinate, $z_{2}=\theta_{2}$

$$
\begin{aligned}
& d_{2} \ddot{\theta}_{2}+d_{3} \cos \left(\theta_{2}-\theta_{1}\right) \ddot{\theta}_{1}+d_{3} \sin \left(\theta_{2}-\theta_{1}\right) \dot{\theta}_{1}^{2}+\sum_{i=1}^{\infty} \ddot{q}_{i}(t) \alpha_{i} \\
& +2 \rho l_{1}\left[\ddot{\theta}_{1} \sin \left(\theta_{2}-\theta_{1}\right)-\dot{\theta}_{1}^{2} \cos \left(\theta_{2}-\theta_{1}\right)\right] \sum_{i=1}^{\infty} q_{i}(t) \frac{\sigma_{i}}{\beta_{i}}+\rho l_{5} \ddot{\theta}_{2} \sum_{i=1}^{\infty} q_{i}^{2}(t) \\
& +2 \rho l_{5} \dot{\theta}_{2} \sum_{i=1}^{\infty} q_{i}(t) \dot{q}_{i}(t)+m_{L} \sum_{i=1}^{\infty} \phi_{i}\left(l_{5}\right) q_{i} \sum_{j=1}^{\infty} \phi_{j}\left(l_{5}\right) q_{j} \ddot{\theta}_{2} \\
& +2 m_{L} \sum_{i=1}^{\infty} \phi_{i}\left(l_{5}\right) q_{i} \sum_{i=1}^{\infty} \phi_{j}\left(l_{5}\right) \dot{q}_{j} \dot{\theta}_{2}+m_{L} l_{1} \sin \left(\theta_{2}-\theta_{1}\right) \sum_{i=1}^{\infty} \phi_{i}\left(l_{5}\right) q_{i} \ddot{\theta}_{1}
\end{aligned}
$$




$$
-m_{L} l_{1} \cos \left(\theta_{2}-\theta_{1}\right) \sum_{i=1}^{\infty} \phi_{i}\left(l_{5}\right) q_{i} \dot{\theta}_{1}^{2}+m_{L}\left(l_{s}+l_{5}\right) \sum_{i=1}^{\infty} \phi_{i}\left(l_{5}\right) \ddot{q}_{i}=\tau_{2} .
$$

For generalized coordinate, $z_{k+2}=q_{k}(k=1,2,3, \ldots \infty)$

$$
\begin{aligned}
- & \frac{2 \sigma_{i}}{\beta_{i}} \rho l_{1} \cos \left(\theta_{2}-\theta_{1}\right) \ddot{\theta}_{1}+\alpha_{i} \ddot{\theta}_{2}+\rho l_{5} \ddot{q}_{i}(t)+\rho l_{5}\left(\omega_{i}^{2}-\dot{\theta}_{2}^{2}\right) q_{i}(t) \\
& -\frac{2 \sigma_{i}}{\beta_{i}} \rho l_{1} \dot{\theta}_{1}^{2} \sin \left(\theta_{2}-\theta_{1}\right)+m_{L} \phi_{i}\left(l_{5}\right)\left(\phi_{1}\left(l_{5}\right) \ddot{q}_{1}+\phi_{2}\left(l_{5}\right) \ddot{q}_{2}\right) \\
& +m_{L}\left(l_{s}+l_{5}\right) \phi_{i}\left(l_{5}\right) \ddot{\theta}_{2}-m_{L} l_{1} \cos \left(\theta_{2}-\theta_{1}\right) \phi_{i}\left(l_{5}\right) \ddot{\theta}_{1} \\
& \left.-m_{L} l_{1} \sin \left(\theta_{2}-\theta_{1}\right) \phi_{i}\left(l_{5}\right) \dot{\theta}_{1}^{2}-m_{L}\left(\phi_{1}\left(l_{5}\right) q_{1}+\phi_{2}\left(l_{5}\right) q_{2}\right) \phi_{i} l_{5}\right) \dot{\theta}_{2}^{2}=0 .
\end{aligned}
$$

The manipulator is actuated by 2 motors and therefore the motor dynamics must be modelled as well. The 2 motors used in this experiment are direct drive brush-less motors. Assuming only friction and viscous damping are present, the dynamic equations of the 2 motors can be written as

$$
\left[\begin{array}{cc}
J_{m 1} & 0 \\
0 & J_{m 2}
\end{array}\right]\left[\begin{array}{l}
\ddot{\theta}_{1} \\
\ddot{\theta}_{2}
\end{array}\right]+\left[\begin{array}{cc}
B_{m l} & 0 \\
0 & B_{m 2}
\end{array}\right]\left[\begin{array}{l}
\dot{\theta}_{1} \\
\dot{\theta}_{2}
\end{array}\right]+\left[\begin{array}{l}
b_{m 1} \operatorname{sgn}\left(\dot{\theta}_{1}\right) \\
b_{m 2} \operatorname{sgn}\left(\dot{\theta}_{2}\right)
\end{array}\right]=\left[\begin{array}{l}
\tau_{m 1} \\
\tau_{m 2}
\end{array}\right]-\left[\begin{array}{l}
\tau_{1} \\
\tau_{2}
\end{array}\right],
$$

where $J_{m_{1}}, J_{m_{2}}$ are the rotor inertia of the motors, $B_{m_{1}}, B_{m_{2}}$ are the viscous damping coefficients, $b_{m_{1}}, b_{m_{2}}$ are the friction coefficients, and $\tau_{m_{1}}, \tau_{m_{2}}$ are the motor torques. From (A11) and (A12), the full dynamic model of the system can be obtained. This is given by

$$
\begin{aligned}
& D(Q) \ddot{Q}+G Q+H(Q, \dot{Q})+\mu\left(\dot{\theta}_{1}, \dot{\theta}_{2}\right)=P T_{m}, \\
& Q=\left[\theta_{1} \theta_{2} q_{1} q_{2} \cdots q_{\infty}\right]^{T}, \quad T_{m}=\left[\tau_{m 1} \tau_{m 2}\right]^{T},
\end{aligned}
$$

$D(Q)=$

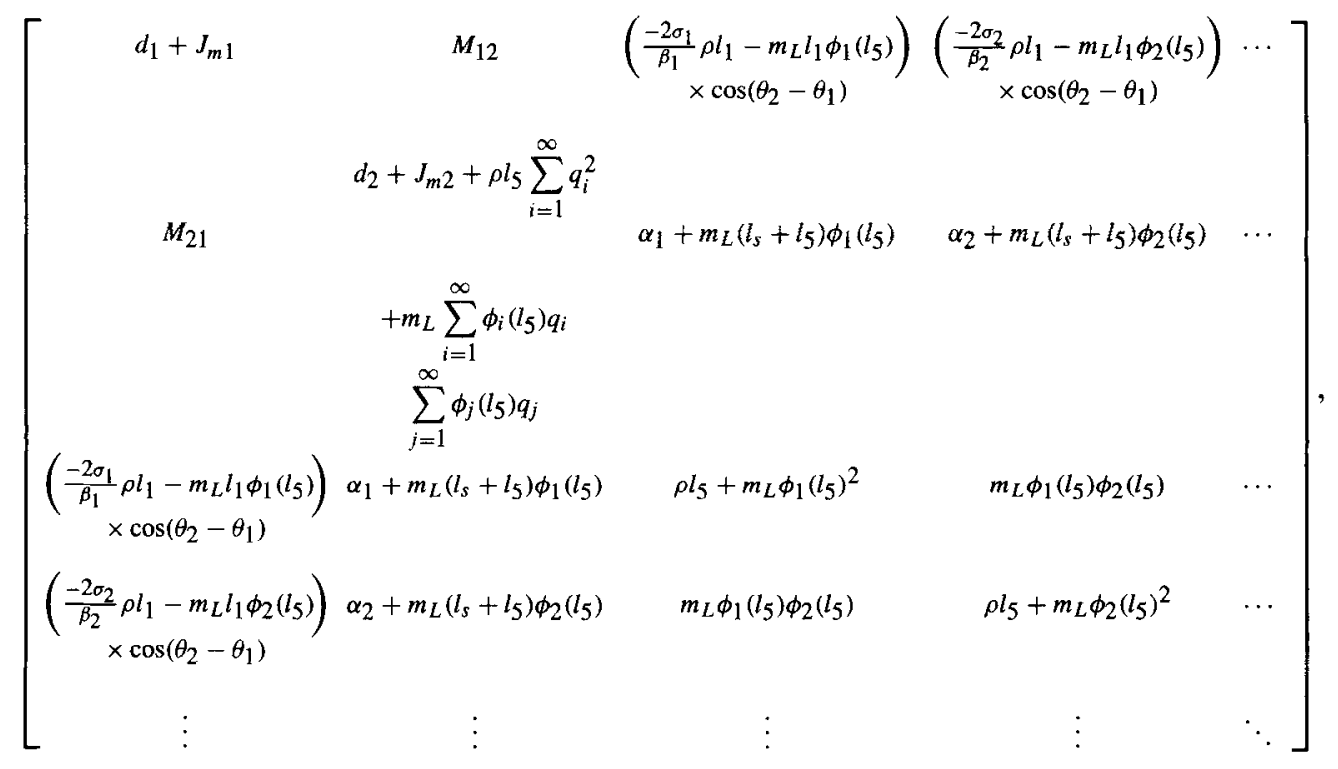




$$
\begin{array}{ccc}
G= & {\left[\begin{array}{ccccc}
0 & 0 & 0 & 0 & \cdots \\
0 & 0 & 0 & 0 & \cdots \\
0 & 0 & \rho l_{5} \omega_{1}^{2} & 0 & \cdots \\
0 & 0 & 0 & \rho l_{5} \omega_{2}^{2} & \cdots \\
\vdots & \vdots & \vdots & \vdots & \ddots
\end{array}\right],} \\
\mu\left(\dot{\theta}_{1}, \dot{\theta}_{2}\right) & =\left[\begin{array}{c}
B_{m 1} \dot{\theta}_{1}+b_{m 1} \operatorname{sgn}\left(\dot{\theta}_{1}\right) \\
B_{m 2} \dot{\theta}_{2}+b_{m 2} \operatorname{sgn}\left(\dot{\theta}_{2}\right) \\
0 \\
0
\end{array}\right], \quad P=\left[\begin{array}{cc}
1 & 0 \\
0 & 1 \\
0 & 0 \\
0 & 0 \\
\vdots & \vdots
\end{array}\right],
\end{array}
$$

$$
H(Q, \dot{Q})=
$$

$$
\left[\begin{array}{rl}
- & d_{3} \sin \left(\theta_{2}-\theta_{1}\right) \dot{\theta}_{2}^{2}+4 \rho l_{1} \dot{\theta}_{2} \sin \left(\theta_{2}-\theta_{1}\right) \sum_{i=1}^{\infty} \frac{\sigma_{i}}{\beta_{i}} \dot{q}_{i} \\
& +2 m_{L} l_{1} \sin \left(\theta_{2}-\theta_{1}\right) \dot{\theta}_{2} \sum_{i=1}^{\infty} \phi_{i}\left(l_{5}\right) \dot{q}_{i} \\
& +\left(2 \rho l_{1} \sum_{i=1}^{\infty} \frac{\sigma_{i}}{\beta_{i}} q_{i}+m_{L} l_{1} \sum_{i=1}^{\infty} \phi_{i}\left(l_{5}\right) q_{i}\right) \cos \left(\theta_{2}-\theta_{1}\right) \dot{\theta}_{2}^{2} \\
d_{3} & \sin \left(\theta_{2}-\theta_{1}\right) \dot{\theta}_{1}^{2}+2 \rho l_{5} \dot{\theta}_{2} \sum_{i=1}^{\infty} q_{i} \dot{q}_{i}+2 m_{L} \sum_{i=1}^{\infty} \phi_{i}\left(l_{5}\right) q_{i} \sum_{j=1}^{\infty} \phi_{j}\left(l_{5}\right) \dot{q}_{j} \dot{\theta}_{2} \\
& -\left(2 \rho l_{1} \sum_{i=1}^{\infty} \frac{\sigma_{i}}{\beta_{i}} q_{i}+m_{L} l_{1} \sum_{i=1}^{\infty} \phi_{i}\left(l_{5}\right) q_{i}\right) \cos \left(\theta_{2}-\theta_{1}\right) \dot{\theta}_{1}^{2} \\
- & \left(2 \frac{\rho_{1}}{\beta_{1}} \rho l_{1}+m_{L} l_{1} \phi_{1}\left(l_{5}\right)\right) \sin \left(\theta_{2}-\theta_{1}\right) \dot{\theta}_{1}^{2} \\
& -\left(\rho_{5} q_{1}+m_{L}\left(\sum_{i=1}^{\infty} \phi_{i}\left(l_{5}\right) q_{i}\right) \phi_{1}\left(l_{5}\right)\right) \dot{\theta}_{2}^{2} \\
& \quad\left(2 \frac{\rho_{2}}{\beta_{2}} \rho l_{1}+m_{L} l_{1} \phi_{2}\left(l_{5}\right)\right) \sin \left(\theta_{2}-\theta_{1}\right) \dot{\theta}_{1}^{2} \\
& -\left(\rho_{5} l_{5} q_{2}+m_{L}\left(\sum_{i=1}^{\infty} \phi_{i}\left(l_{5}\right) q_{i}\right) \phi_{2}\left(l_{5}\right)\right) \dot{\theta}_{2}^{2} \\
d_{1}= & I_{1}+m_{1} l_{c 1}^{2}+I_{3}+m_{3} l_{c 3}^{2}+m_{4} l_{1}^{2}+m_{5} l_{1}^{2}+m_{c} l_{1}^{2}+m_{L} l_{1}^{2} \\
d_{2}= & I_{2}+m_{2} l_{c 2}^{2}+I_{4}+m_{3} l_{2}^{2}+m_{4} l_{c 4}^{2}+\frac{1}{3} m_{5} l_{5}^{2}+m_{5} l_{s}^{2} \\
& +m_{L}\left(l_{s}+l_{5}\right)^{2},
\end{array}\right]
$$




$$
\begin{aligned}
d_{3} & =m_{3} l_{2} l_{c 3}-\frac{1}{2} m_{5} l_{1} l_{5}+m_{4} l_{1} l_{c 4}-m_{5} l_{s} l_{1}-m_{L} l_{1}\left(l_{s}+l_{5}\right)+m_{c} l_{1} l_{c} \\
M_{12} & =M_{21}=d_{3} \cos \left(\theta_{2}-\theta_{1}\right) \\
& +\left[2 \rho l_{1} \sum_{i=1}^{\infty} \frac{\sigma_{i}}{\beta_{i}} q_{i}+m_{L} l_{1} \sum_{i=1}^{\infty} \phi_{i}\left(l_{5}\right) q_{i}\right] \sin \left(\theta_{2}-\theta_{1}\right) \\
\alpha_{i} & =2 \rho\left(l_{s} \frac{\sigma_{i}}{\beta_{i}}+\frac{1}{\beta_{i}^{2}}\right)
\end{aligned}
$$

\section{References}

Aoustin Y et al 1994 Experimental results for the end-effector control of a single flexible robotic arm. IEEE Trans. Control Syst. Technol. 2: 371-381

Banavar R N, Dominic P 1995 An LQG/H-infinity controller for a flexible manipulator. IEEE Trans. Control Syst. Technol. 3: 409-416

Book W J 1984 Recursive Langrangian dynamics of flexible manipulor arms. Int. J. Robotics Res. 3: $87-101$

Cannon R H, Schmitz E J 1984 Initial experiments on the end-point control of a flexible one-link robot. Int. J. Robotics Res. 3: 62-75

Craig R R Jr (ed.) 1981 Structural dynamics: An introduction to computer methods (New York: John Wiley \& Sons)

De Luca A, Siciliano B 1993 Inversion based nonlinear control of robot arms with flexible links. AIAA J. Guidance Control Dyn. 16: 1169-1176

Gross E, Tomizuka M 1994 Experimental flexible beam tip tracking with a truncated series approximation to uncancellable inverse dynamics. IEEE Trans. Control Syst. Technol. 2: 382391

Hastings G G, Books W J 1986 Verification of a linear dynamic model for flexible robotic manipulators. Proc. of the IEEE Conference on Robotics and Automation (Washington, DC: IEEE Comput. Soc. Press) pp 1024-1029

Khorrami F, Jain S, Tzes A 1994 Experiments on rigid body-based controllers with input preshaping for a two-link flexible manipulator. IEEE Trans. Robotics Autom. 10: 55-64

Lin L C, Yih T W 1996 Rigid model-based neural network control of flexible-link manipulators. IEEE Trans. Robotics Autom. 12: 595-601

Matsuno F, Fukushima S 1987 Feedback control of a flexible manipulator with a parallel drive mechanism. Int. J. Robotics Res. 6: 76-84

Mills J K, Lokhorst D M 1993 Control of robotic manipulators during general task execution A discontinuous control approach. Int. J. Robotics Res. 12: 146-163

Moudgal V G, Passino K M, Yurkovich S 1994 Rule-based control for a flexible link robot. IEEE Trans. Control Syst. Technol. 2: 392-405

Sakawa Y, Matsuno F 1986 Modelling and control of a flexible manipulator with a parallel drive mechanism. Int. J. Control 44: 299-313

Siciliano B, Book W J 1988 A singular perturbation approach to control of lightweight flexible manipulators. Int. J. Robotics Res. 7: 79-90

Siciliano B, Prasad J V R, Calise A J 1992 Output feedback two-time-scale control of multilink flexible arms. ASME J. Dyn. Syst. Meas. Control 114: 70-77

Vandergrift M W, Lewis F L, Zhu S Q 1994 Flexible-link robot arm control by a feedback linearization/singular perturbation approach. J. Robotic Syst. 11: 591-603 
Wang D, Vidyasagar M 1991 Control of a class of manipulators with a single flexible link - part 1: Feedback linearization. ASME J. Dyn. Syst. Meas. Control 113:655-661

Zhu S Q, Lewis F L, Hunt L 1994 Robust stabilization of the internal dynamics of flexible robots without measuring the velocity of the deflection. Proc. IEEE Conf. Decision and Control, Orlando, pp 1811-1817 